Pacific Journal of Mathematic 


\title{
EXTENSION OF UNIFORMLY CONTINUOUS TRANSFORMATIONS AND HYPERCONVEX METRIC SPACES
}

\author{
N. Aronszajn AND P. PanitchPakdi
}

Introduction. The results of the present paper combine the research done by the first author mainly in 1929-1930 (which was never published) and the results of the thesis presented by the second author at the University of Kansas, 1955.

The principal topic of the first two sections is the following: can a uniformly continuous transformation $T$ of a metric space $\mathscr{D}$ into a metric space $\mathscr{F}$ be extended with conservation of modulus of continuity to any larger metric space $\mathscr{E}$ containing $\mathscr{D}$ metrically so that the range is still contained in $\mathscr{F}$ ? In $\S 1$ we show (Theorem 2) that for the possibility of such unlimited extension of $T$ it is necessary that the minimal modulus of continuity of $T$ satisfy a condition which is proved in Theorem 1 to be necessary and sufficient for the existence of a subadditive modulus of continuity for $T$. In $\S 2$ the transformations $T$ are restricted to be those with a subadditive modulus of continuity. The main result of this section is that a necessary and sufficient condition that there exist an unlimited extension of any transformation $T$ into a space $\mathscr{F}$ with conservation of a subadditive modulus of continuity $\delta(\varepsilon)$ is that $\mathscr{F}$ be hyperconvex ${ }^{1}$ (see Definition 1 of $\S 2$ ). The $\mathfrak{m}$-hyperconvexity is introduced for any cardinal $\mathfrak{m} \geqq 3$, which is a weaker property than hyperconvexity.

In $\S 3$ the properties of hyperconvex (or m-hyperconvex) spaces and subsets of metric spaces are investigated. As a useful tool the notion of almost $m$-hyperconvexity is introduced; it is slightly weakar than m-hyperconvexity. The main results of this section are the following: ith-hyperconvexity implies completeness for $\mathfrak{m}>\boldsymbol{h}_{0}$ (Theorem $1^{\prime}$ ); almost $\mathfrak{m}$-hyperconvexity and completeness imply $\mathfrak{m}$-hyperconvexity for $\mathfrak{m} \geqq \widehat{t}_{0}$ (Theorem 4). In any complete metric space the class of all n-hyperconvex subsets is considered as a subset of the class of all closed subsets provided with the well known metric introduced by Hausdorff. It is proved that $\mathfrak{m}$-hyperconvex subsets form a closed set in the class of all closed subsets (Theorem 5). The topological properties of hyperconvex spaces are then investigated. It is proved that every hyperconvex space is a generalized absolute retract.

In $\S 4$ the hyperconvex Banach spaces are considered and a direct

Received June 1, 1955. This work was done under Research Grant NSF-G1126 with the National Science Foundation.

1 This suggestive term "hyperconvex" was proposed to the authors by A. H. Kruse. 
proof is given of a conjecture of $\mathrm{L}$. Nachbin that the closed unit sphere of a hyperconvex Banach space possesses extreme points ${ }^{2}$.

In $\S 5$ the Banach spaces $\mathscr{C}(\mathscr{C})$ of continuous real functions on compact Hausdorff spaces $\mathscr{C}$ are considered from the point of view of m-hyperconvexity. In Theorem 2 a necessary and sufficient topological condition $\left(Q_{\mathfrak{m}}\right)$ for the space $\mathscr{C}$ is given in order that $\mathscr{C}(\mathscr{K})$ be $\mathfrak{m}^{-}$ hyperconvex.

In the final $\S 6$ relevant examples are given illustrating the developments of previous sections. Also several unsolved problems are stated.

1. Moduli of uniform continuity. We shall call an extended valued nonnegative function $\delta(\varepsilon), 0<\varepsilon<\infty$, a modulus of uniform continuity, or briefly a modulus of continuity, if it is nondecreasing and converges to 0 as $\varepsilon$ goes to 0 .

If $T$ is a transformation of a metric space $\mathscr{O}$ into a metric space $\mathscr{F}$, we call $\delta(\varepsilon)$ a modulus of continuity of $T$ if, for $x, y$ in $\mathscr{D}$ and $\rho(x, y) \leqq \varepsilon$,

$$
\rho_{1}(T(x), T(y)) \leqq \delta(\varepsilon),
$$

where $\rho$ and $\rho_{1}$ denote the metrics of $\mathscr{D}$ and $\mathscr{F}$ respectively.

If a transformation $T$ has some modulus of continuity, it is uniformly continuous.

For each uniformly continuous transformation $T$ there exists a minimal modulus of continuity $\delta_{T}(\varepsilon)$ defined as follows:

$$
\delta_{P}(\varepsilon)=\sup \left[\rho_{1}(T(x), T(y)) ; x, y \varepsilon \mathscr{D}, \rho(x, y) \leqq \varepsilon\right] .
$$

Every other modulus of continuity of $T$ satisfies the obvious relation $\delta_{T}(\varepsilon) \leqq \delta(\varepsilon)$ for all $\varepsilon>0$.

A modulus of continuity is called subadditive if

$$
\delta\left(\varepsilon_{1}+\varepsilon_{2}\right) \leqq \delta\left(\varepsilon_{1}\right)+\delta\left(\varepsilon_{2}\right)
$$

for any $\varepsilon_{1}>0$ and $\varepsilon_{2}>0$.

THEOREM 1. In order that there exist a subadditive modulus of continuity majorating a given modulus of continuity $\delta(\varepsilon)$, it is necessary and sufficient that

$$
\limsup _{\varepsilon \rightarrow \infty} \frac{\delta(\varepsilon)}{\varepsilon}<\infty
$$

2 This conjecture was already indirectly proved by J. L. Kelley [11]. Numbers in square brackets refer to the references at the end of the paper. 
Proof. If $\delta_{0}(\varepsilon)$ is a subadditive modulus of continuity majorating $\delta(\varepsilon)$, then for some $\varepsilon_{0}>0$ such that $\delta_{0}\left(\varepsilon_{0}\right)<\infty$ and all $\varepsilon>\varepsilon_{0}$, we have

$$
\begin{aligned}
& \limsup _{\varepsilon \rightarrow \infty} \frac{\delta(\varepsilon)}{\varepsilon} \leqq \limsup _{\varepsilon \rightarrow \infty} \frac{\delta_{0}\left(\varepsilon_{0}\left[\begin{array}{c}
\varepsilon \\
-\varepsilon_{0}
\end{array}\right]+\varepsilon_{0}\right)}{\varepsilon} \\
& \leqq \limsup _{\varepsilon \rightarrow \infty} \frac{\left[\begin{array}{c}
\varepsilon \\
\varepsilon_{0}
\end{array}\right]+1}{\varepsilon} \delta\left(\varepsilon_{0}\right) \\
& =\frac{\delta\left(\varepsilon_{0}\right)}{\varepsilon_{0}}<\infty,
\end{aligned}
$$

where $[a]$ denotes the greatest integer in $a$. Hence the condition is necessary.

To prove the sufficiency of the condition we observe first that since $\delta$ satisfies (1.4), there exist positive numbers $\varepsilon_{1}$ and $M$ such that $\delta(\varepsilon) \leqq M \varepsilon$ for $\varepsilon \geqq \varepsilon_{1}$, and hence, for every $\alpha$ with $0<\alpha<\varepsilon_{1}$,

$$
\delta(\varepsilon) \leqq \delta(\alpha)+\frac{\varepsilon_{1}}{\alpha} M \varepsilon \quad \text { for all } \varepsilon>0 .
$$

This is easy to check, by considering $\varepsilon$ separately in the intervals $(0 ; \alpha]$, $\left(\alpha ; \varepsilon_{1}\right]$, and $\left(\varepsilon_{1} ; \infty\right)$.

Define now the function $\delta_{0}(\varepsilon)$ for all $\varepsilon \geq 0$ by

$$
\delta_{0}(\varepsilon)=\inf _{0<\alpha<\varepsilon_{1}}\left[\delta(\alpha)+{ }^{\varepsilon_{1}} M \varepsilon\right]
$$

This function is obviously finite, nonnegative, nondecreasing, and satisfies $\delta(\varepsilon) \leqq \delta_{0}(\varepsilon)$. Furthermore, for $\varepsilon<\varepsilon_{1}^{2}, \quad \delta_{0}(\varepsilon) \leqq \delta(V \varepsilon)+\varepsilon_{1} M V \varepsilon$, hence $\lim _{\varepsilon \searrow 0} \delta_{0}(\varepsilon)=\delta_{0}(0)=0$. Finally, since $\delta_{0}(\varepsilon)$ is the inf. of linear functions it is concave (that is, $\delta_{0}\left(\beta^{\prime} \varepsilon^{\prime}+\beta^{\prime \prime} \varepsilon^{\prime \prime}\right) \geq \beta^{\prime} \delta_{0}\left(\varepsilon^{\prime}\right)+\beta^{\prime \prime} \delta_{0}\left(\varepsilon^{\prime \prime}\right)$ for all $\varepsilon^{\prime}, \varepsilon^{\prime \prime}, \beta^{\prime}$, and $\beta^{\prime \prime}$ nonnegative with $\left.\beta^{\prime}+\beta^{\prime \prime}=1\right)$. From this it follows that $\delta_{0}(\varepsilon)$ is continuous and subadditive ${ }^{3}$.

A metric space $\mathscr{E}$ is called convex $x^{4}$ if for any two of its points $x$, $y, x \neq y$, and for some $\alpha, \beta$ with $\rho(x, y)=\alpha+\beta, \alpha>0, \beta>0$, there exists a point $z$ in $\mathscr{E}$ with $\rho(x, z)=\alpha$ and $\rho(z, y)=\beta$. If such a point $z$ exists for any decomposition $\rho(x, y)=\alpha+\beta, \alpha>0, \beta>0$, then $\mathscr{E}$ is called totally convex ${ }^{5}$.

REMARK 1. If the domain $\mathscr{Y}$ of a transformation $T$ is a totally convex space, then the minimal modulus of continuity is subadditive.

3 See, for example, E. Hille [9].

${ }^{4}$ See K. Menger [13].

$\checkmark$ See K. Menger [13]. 
In fact, for some given $\varepsilon_{1}>0, \varepsilon_{2}>0$ and for every arbitrarily small number $\eta>0$, there exist points $x, y$ in $\mathscr{D}$ such that $\rho(x, y) \leqq \varepsilon_{1}+\varepsilon_{2}$ and $\rho_{1}(T(x), T(y))>\delta_{T}\left(\varepsilon_{1}+\varepsilon_{2}\right)-\eta$. For $x$ and $y$ we can choose a point $z$ such that

$$
\rho(x, z)=\frac{\varepsilon_{1}}{\varepsilon_{1}+\varepsilon_{2}} \rho(x, y) \leqq \varepsilon_{1}
$$

and

$$
\rho(z, y)=\frac{\varepsilon_{2}}{\varepsilon_{1}+\varepsilon_{2}} \rho(x, y) \leqq \varepsilon_{2}
$$

From this we get

$$
\begin{aligned}
\delta_{T}\left(\varepsilon_{1}+\varepsilon_{2}\right)-\eta<\rho_{1}(T(x), T(y)) & \leqq \rho_{1}(T(x), T(z))+\rho_{1}(T(z), T(y)) \\
& \leqq \delta_{T}\left(\varepsilon_{1}\right)+\delta_{T}\left(\varepsilon_{2}\right) .
\end{aligned}
$$

Since $\eta$ is arbitrary, our assertion follows.

We will say that a transformation $T$ of a metric space $\mathscr{Y}$ into a metric space $\mathscr{F}$ has the unlimited uniform extension property with respect to $\mathscr{F}$ if for any metric space $\mathscr{E}$ containing $\mathscr{D}$ metrically ${ }^{6}$, there exists a uniformly continuous extension of $T$ to the whole of $\mathscr{E}$ with range in $\mathscr{F}$. Clearly if $T$ has this property, then it must be itself uniformly continuous.

THEOREM 2. In order that a transformation $T$ of $\mathscr{D}$ into $\mathscr{F}$ have the unlimited uniform extension property with respect to $\mathscr{F}$ it is necessary that its minimal modulus of continuity $\delta_{T}(\varepsilon)$ satisfy the property (1.4) of Theorem 1.

Proof. Suppose that the property (1.4) is not true for $\delta_{T}(\varepsilon)$. Then there exists a sequence of real numbers $\left\{\varepsilon_{n}\right\}$ such that $\varepsilon_{n}>n$ and $\frac{\delta_{T}\left(\varepsilon_{n}\right)}{\varepsilon_{n}}>n$, $n=1,2, \cdots$. Therefore by the definition of $\delta_{T}(\varepsilon)$, there exist $x_{n}, y_{n}$ in $\mathscr{D}$ such that $\alpha_{n}=\rho\left(x_{n}, y_{n}\right) \leqq \varepsilon_{n}$ and $\rho_{1}\left(T\left(x_{n}\right), T\left(y_{n}\right)\right)>n \varepsilon_{n}$.

Form a space $\mathscr{E}$ from the points of $\mathscr{D}$ and the points of the form $\left(n, \xi_{n}\right), 0<\xi_{n}<\alpha_{n}, n=1,2, \cdots$. Define the metric $\rho^{\prime}$ on $\mathscr{E}$ in the following way:

(1) $x, y$ in $\mathscr{D}, \rho^{\prime}(x, y)=\rho(x, y)$.

(2) $x$ in $\mathscr{D}, y=\left(n, \xi_{n}\right)$,

$$
\rho^{\prime}(x, y)=\rho^{\prime}(y, x)=\min \left[\rho\left(x, x_{n}\right)+\xi_{n}, \rho\left(x, y_{n}\right)+\alpha_{n}-\xi_{n}\right],
$$

${ }^{6} \mathscr{D}$ is contained in $\mathscr{E}$ metrically if it is a subset of $\mathscr{E}$ and the metric of $\mathscr{E}$, for the points of $\mathscr{D}$, coincides with the original metric of $\mathscr{D}$. 
(3) $x=\left(n, \xi_{n}\right), \quad y=\left(n, \eta_{n}\right), \quad \rho^{\prime}(x, y)=\rho^{\prime}(y, x)=\left|\xi_{n}-\eta_{n}\right|$,

(4) $x=\left(m, \xi_{m}\right), y=\left(n, \eta_{n}\right), \rho^{\prime}(x, y)=\rho^{\prime}(y, x)$

$=\min \left[\rho^{\prime}\left(x_{m}, y\right)+\xi_{m}, \rho^{\prime}\left(y_{m}, y\right)+\alpha_{m}-\xi_{m}\right]$

$=\min \left[\rho^{\prime}\left(x_{n}, x\right)+\eta_{n}, \rho^{\prime}\left(y_{n}, x\right)+\alpha_{n}-\eta_{n}\right]$.

The last equality in (4) can easily be checked, and also there is no difficulty in verifying that $\rho^{\prime}$ satisfies all conditions for a metric in the space $\mathscr{E}$.

Suppose that there exists a uniformly continuous extension $\tilde{T}$ of $T$ to the whole of $\mathscr{E}$ with range in $\mathscr{F}$. Then let $\delta(\varepsilon)$ be a modulus of continuity for $\tilde{T}$, and let $\varepsilon^{\prime}$ be a positive number such that $\delta\left(\varepsilon^{\prime}\right)<\infty$. Denote $\left[\begin{array}{c}\varepsilon_{n} \\ \varepsilon^{\prime}\end{array}\right]+1$ by $m_{n}$. Consider now the points of $\mathscr{E}$ of the following type :

$$
\left(n, \frac{\alpha_{n}}{m_{n}} i\right)=x_{n, i}, \quad i=1,2, \cdots, m_{n}-1, \quad x_{n, 0}=x_{n}
$$

and

$$
x_{n, m_{n}}=y_{n}
$$

By definitions (2) and (3), we have

$$
\rho^{\prime}\left(x_{n, i}, x_{n, i+1}\right)=\frac{\alpha_{n}}{m_{n}} \leqq \frac{\varepsilon_{n}}{\frac{\varepsilon_{n}}{\varepsilon^{\prime}}}=\varepsilon^{\prime} .
$$

Therefore

$$
\rho_{1}\left(T\left(x_{n}\right), T\left(y_{n}\right)\right) \leqq \sum_{i=0}^{m_{n}-1} \rho_{1}\left(T\left(x_{n, i}\right), T\left(x_{n, i+1}\right)\right) \leqq m_{n} \delta\left(\varepsilon^{\prime}\right) .
$$

On the other hand, this means that

$$
n \varepsilon_{n} \leqq m_{n} \delta\left(\varepsilon^{\prime}\right) \leqq \delta\left(\varepsilon^{\prime}\right)\left(\begin{array}{c}
\varepsilon_{n}+1 \\
\varepsilon^{\prime}
\end{array}\right)
$$

and so for $n \rightarrow \infty$,

$$
n \leqq \delta\left(\varepsilon^{\prime}\right)\left(\frac{1}{\varepsilon^{\prime}}+\frac{1}{\varepsilon_{n}}\right)<\delta\left(\varepsilon^{\prime}\right)\left(\frac{1}{\varepsilon^{\prime}}+\frac{1}{n}\right),
$$

which is impossible.

2. Hyperconvex spaces. Extension of uniformly continuous transformations. The main purpose of this section will be the study of 
extension of uniformly continuous transformations with conservation of moduli of continuity ; in particular, we shall be interested in the property of the space $\mathscr{F}$ which guarantees that every uniformly continuous transformation $T$ with range in $\mathscr{F}$ has the unlimited extension property with respect to $\mathscr{T}$, the extension conserving a given modulus of continuity $\delta(\varepsilon)$ of $T$.

Theorem 2 of the previous section shows that if this requirement is satisfied, the minimal modulus of continuity of $T$ must fulfill condition (1.4). Then Theorem 1 says that we can choose for $T$ a subadditive modulus of continuity. We should therefore correct the statement of our problem by restricting it to any transfomation with range in $\mathscr{F}$ which admits a subadditive modulus of continuity $\delta(\varepsilon)$. We wish all such transformations to have the unlimited extension property with respect to $\mathscr{F}$, their extensions admitting the same modulus $\delta(\varepsilon)$. In order to formulate the necessary and sufficient condition for $\mathscr{F}$ so that our requirements be satisfied, we are going to introduce some definitions.

In the following definitions, we denote by $m$ any fixed cardinal number $\geq 3$.

Definition 1. A metric space $\mathscr{E}$ will be called hyperconvex (or

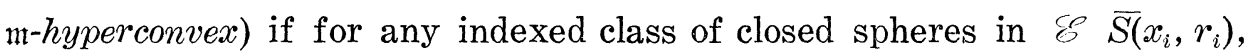
$i \in I$ (or for any such class with card $(I)<\mathrm{m}$ ), satisfying the condition that $\rho\left(x_{i}, x_{j}\right) \leqq r_{i}+r_{j}$ for all $i, j$ in $I$, the intersection $\bigcap_{i \in I} \overline{S(}\left(x_{i}, r_{i}\right)$ is not empty.

It is clear that hyperconvexity is stronger than $m$-hyperconvexity, which is stronger than $\mathfrak{n}$-hyperconvexity if $\mathfrak{n}<\mathfrak{n}$. For $\mathfrak{m}=3$, it is easy to see that 3-hyperconvexity is equivalent to total convexity. (In fact, if the space is totally convex and if $\rho\left(x_{1}, x_{2}\right) \leqq r_{1}+r_{2}$, then the point $z$ which realizes the distances $\rho(x, z)=\frac{r_{1}}{r_{1}+r_{2}} \rho\left(x_{1}, x_{2}\right)$ and $\rho\left(x_{2}, z\right)=\frac{r_{2}}{r_{1}+r_{2}} \rho\left(x_{1}, x_{2}\right)$, will lie in $\left.\overline{S(}\left(x_{1}, r_{1}\right) \cap \overline{S(} x_{2}, r_{2}\right)$. On the other hand, if the space is $3-$ hyperconvex, then for the decomposition $\rho\left(x_{1}, x_{2}\right)=\alpha+\beta, \alpha>0, \beta>0$, we take any $z$ in $\left.\overline{S(} x_{1}, \alpha\right) \cap \bar{S}\left(x_{2}, \beta\right)$, and therefore $\rho\left(x_{1}, z\right) \leqq \alpha, \rho\left(x_{2}, z\right) \leqq \beta$, which are in fact equalities because of $\rho\left(x_{1}, z\right)+\rho\left(x_{2}, z\right) \geq \rho\left(x_{1}, x_{2}\right)=\alpha+\beta$.) This is the justification of the term hyperconvexity.

Definition 2. A metric space is called $\mathrm{m}$-separable if it contains a dense subset of cardinal $<\mathrm{m}$.

It is obvious that $\mathfrak{m}$-separability is weaker than $\mathfrak{n}$-separability if $\mathfrak{n}<\mathfrak{m}$, and $\boldsymbol{H}_{1}$-separability coincides with the usual separability. $\boldsymbol{N}_{0}-$ separability means that the metric space is a finite set, and $\mathfrak{m}$-separability for a finite cardinal $\mathfrak{m}$ means that the space contains at most $\mathfrak{m}-1$ points. 
We shall prove first a few statements about spaces having the above properties.

THEOREM 1. If the space $\mathscr{E}$ is m-hyperconvex and at the same time $\mathfrak{m}$-separable, then it is hyperconvex.

Proof. Consider an arbitrary indexed family of spheres $\overline{S(}\left(x_{i}, r_{i}\right)$, $i \in I$, satisfying the requirements of Definition 1 , that is, $\rho\left(x_{i}, x_{j}\right) \leqq r_{i}+r_{\text {, }}$ for $i, j \in I$. Let $\left\{p_{k}\right\}, k \in K$ with card $(K)<m$, be an indexed set of points, which is dense in $\mathscr{E}$. Put

(2.1) $r_{k}^{\prime}=$ the infimum of all $r>0$ such that there exists $i \in I$ with $\left.\overline{S(}\left(p_{k}, r\right) \supset \overline{S(} x_{i}, r_{i}\right)$.

The class of spheres $\left.\overline{S(} p_{k}, r_{k}^{\prime}\right), k \in K$, satisfies the requirement for $\mathfrak{m}$ hyperconvexity. In fact, card $(K)<\mathfrak{m}$. Take any two indices $k, l$ in $K$ and an arbitrary $\varepsilon>0$. By (2.1) there exist $i \in I, j \in I$ such that

$$
\overline{S(}\left(p_{k}, r_{k}^{\prime}+\varepsilon\right) \supset \bar{S}\left(x_{i}, r_{i}\right)
$$

and

$$
\left.\overline{S(} p_{l}, r_{l}^{\prime}+\varepsilon\right) \supset \bar{S}\left(x_{j}, r_{j}\right) \text {. }
$$

The two spheres $\left.\overline{S(} x_{i}, r_{i}\right)$ and $\left.\overline{S(} x_{j}, r_{j}\right)$ satisfy the requirement of mhyperconvexity. Therefore there exists a point $q$ in

$$
\bar{S}\left(x_{i}, r_{i}\right) \cap \bar{S}\left(x_{j}, r_{j}\right) ;
$$

a fortiori, $q$ is in

$$
\left.\left.\overline{S(} p_{k}, r_{k}^{\prime}+\varepsilon\right) \cap \overline{S(} p_{l}, r_{l}^{\prime}+\varepsilon\right)
$$

That means

$$
\rho\left(p_{k}, p_{l}\right) \leqq \rho\left(p_{k}, q\right)+\rho\left(p_{l}, q\right) \leqq r_{k}^{\prime}+r_{l}^{\prime}+2 \varepsilon .
$$

Since $\varepsilon$ is arbitrary, we obtain the requirement for $\mathfrak{m}$-hyperconvexity for $\left\{\bar{S}\left(p_{k}, r_{k}^{\prime}\right)\right\}, k \in K$. It follows that there is a point $x$ in $\bigcap_{k \in K} \overline{S(}\left(p_{k}, r_{k}^{\prime}\right)$. What remains to be proved is that for every $i$ in $I, x \in \bar{S}\left(x_{i}, r_{i}\right)$, that is, $\rho\left(x, x_{i}\right) \leqq r_{i}$. For this purpose take an arbitrary $\varepsilon>0$. By density of the set $\left\{p_{k}\right\}, k \in K$, there exists a point $p_{k}$ for each $x_{i}$ such that

$$
\rho\left(x_{i}, p_{k}\right)<\varepsilon \text {. }
$$

Hence

$$
\bar{S}\left(p_{k}, r_{i}+\varepsilon\right) \supset \bar{S}\left(x_{i}, r_{i}\right)
$$




\section{Therefore}

$$
r_{k}^{\prime} \leqq r_{i}+\varepsilon,
$$

and

$$
\rho\left(x, x_{i}\right) \leqq \rho\left(x, p_{k}\right)+\rho\left(p_{k}, x_{i}\right)<r_{k}^{\prime}+\varepsilon \leqq r_{i}^{\prime}+2 \varepsilon .
$$

Since $\varepsilon$ is arbitrary, the proof is complete.

REMARK 1. The above theorem means that for $\mathrm{m}$-separable spaces, $m$-hyperconvexity is equivalent to hyperconvexity. For $\mathfrak{m} \leqq \mathrm{x}_{0}$ the theorem is completely trivial since $\mathfrak{m}$-separability means then that the space has a finite number of points, whereas m-hyperconvexity implies total convexity, which is impossible in a finite set except when the set is reduced to a single point. For such a set clearly all our properties are trivially satisfied.

REMARK 2. Since every space $\mathscr{E}$ is $\mathfrak{m}$-separable for $\mathrm{m}>$ card $(\mathscr{E})$, it follows that hyperconvexity of $\mathscr{E}$ is equivalent to $\mathfrak{m}$-hyperconvexity of $\mathscr{E}$ for any $m>$ card $(\mathscr{E})$.

We give now a simple and almost obvious lemma, which we shall need later.

LemMA. If a metric space is $(\mathfrak{m}+1)$-separable, $\mathfrak{m} \geq 1$, then any proper subset of it is m-separable.

Proof. In fact, take first $\mathfrak{m}$ to be finite. Then $(\mathfrak{m}+1)$-separability means that the space has at most $m$ points and any of its proper subsets has at most $\mathfrak{m}-1$ points. Therefore it is $\mathfrak{m}$-separable. If $\mathfrak{m} \geq \mathfrak{\mathfrak { N } _ { 0 }}$, then $\mathfrak{m}+1=\mathfrak{m}$. Let $\left\{p_{i}\right\}, i \in I$, be an indexed set dense in $\mathscr{E}$ with card $(I)<\mathfrak{m}$. For each $i$ in $I$, consider in a proper subset $\mathscr{D}$ of $\mathscr{E}$ a sequence $\left\{p_{i, n}\right\}, n \in N=$ the set of positive integers, such that $\lim _{n \rightarrow \infty} \rho\left(p_{i}, p_{i, n}\right)=\rho\left(p_{i}, \mathscr{D}\right)^{7}$. It is easily checked that the set $\left\{p_{i, n}\right\},(i, n) \in I \times N$, is dense in $\mathscr{D}$ and card $(I \times N)<\mathfrak{m} \cdot \aleph_{0}=\mathfrak{m}$.

We can now consider our problem of unlimited extension of a uniformly continuous transformation with range in a given metric space F with metric $\rho_{1}$. Among the subadditive moduli of continuity, especially important is the one given by $\delta(\varepsilon)=\varepsilon$. If a transformation admits of this modulus of continuity, we will call it a contraction. The condition we shall give in what follows will turn out to be necessary already if we restrict the transformations to contractions, and suflicient if we accept a transformation with arbitrary subadditive modulus of continuity.

${ }^{7} \rho\left(p_{i}, \mathscr{D}\right)$ is the shortest distance between $p_{i}$ and $\mathscr{D}$ and may be zero. 
Therefore it will be convenient to give the two types of theorems separately.

We use the notation $T<\tilde{T}$, or $\tilde{T} \succ T$, for two transformations $T$ and $\tilde{T}$ if $\tilde{T}$ is an extension of $T$.

THEOREM 2. Let $\mathscr{F}$ be a metric space and $\mathfrak{m}$ be a cardinal number $\geq 3$. If for every contraction $T$ with some domain $\mathscr{D}$ and range $T(\mathscr{D})$ in $\mathscr{F}$, and for any $(\mathfrak{m}+1)$-separable $e^{8}$ metric space $\mathscr{E}$ containing $\mathscr{D}$ metrically, there exists a contraction $\tilde{T}$ of $\mathscr{E}$ into $\mathscr{F}$ such that $\tilde{T}>T$, then the space $\mathscr{F}$ is $\mathfrak{m}$-hyperconvex.

Proof. We start by proving that $\mathscr{F}$ is totally convex, that is, 3hyperconvex. In fact, consider two spheres $\bar{S}\left(x_{1}, r_{1}\right)$ and $\left.\overline{S(} x_{2}, r_{2}\right)$ with $\rho_{1}\left(x_{1}, x_{2}\right) \leqq r_{1}+r_{2}$. We may assume $x_{1} \neq x_{2}$ otherwise there is nothing to prove). Let $T$ be the identity transformation defined on $\mathscr{D}=\left(x_{1}, x_{2}\right)$. As the space $\mathscr{E}$ consider $\mathscr{D} \cup(y), y$ being a point not in $\mathscr{F}$, and define the metric $\rho$ on $\mathscr{E}$ by putting

$$
\begin{aligned}
& \rho\left(x_{2}, x_{1}\right)=\rho\left(x_{1}, x_{2}\right)=\rho_{1}\left(x_{1}, x_{2}\right), \\
& \rho\left(x_{1}, x_{1}\right)=\rho\left(x_{2}, x_{2}\right)=0, \quad \rho(y, y)=0, \\
& \rho\left(x_{1}, y\right)=\rho\left(y, x_{1}\right)=\frac{r_{1}}{r_{1}+r_{2}} \rho_{1}\left(x_{1}, x_{2}\right), \\
& \rho\left(x_{2}, y\right)=\rho\left(y, x_{2}\right)=\frac{r_{2}}{r_{1}+r_{2}} \rho_{1}\left(x_{1}, x_{2}\right) .
\end{aligned}
$$

It is clear that $\mathscr{E}$ is a metric space and that it is certainly $(\mathfrak{m}+1)$ separable. By hypothesis, there exists an extension $\tilde{T}>T$, which is a contraction of $\mathscr{E}$ into $\mathscr{F}$. That means

$$
\rho_{1}\left(\tilde{T}(y), x_{1}\right)=\rho_{1}\left(\tilde{T}(y), \tilde{T}\left(x_{1}\right)\right) \leqq \rho\left(y, x_{1}\right)=\frac{r_{1}}{r_{1}+r_{2}} \rho_{1}\left(x_{1}, x_{2}\right) \leqq r_{1},
$$

and similarly

$$
\rho_{1}\left(\tilde{T}(y), x_{2}\right) \leqq \frac{r_{2}}{r_{1}+r_{2}} \rho_{1}\left(x, x_{2}\right) \leqq r_{2},
$$

which shows that $\overline{S(}\left(x_{1}, r_{1}\right) \cap \bar{S}\left(x_{2}, r_{2}\right) \neq 0$.

Take now an indexed class of spheres in $\left.\mathscr{F} \overline{S(} x_{i}, r_{i}\right), i \in I$, card $(I)<\mathfrak{m}$, with $\rho_{1}\left(x_{i}, x_{j}\right) \leqq r_{i}+r_{j}$ for $i, j \in I$. We want to prove that $\bigcap_{i \in I} \overline{S(}\left(x_{i}, r_{i}\right) \neq 0$. Consider therefore the set $\mathscr{D}=\left\{x_{i}\right\}, i \in I$; $\operatorname{card}(\mathscr{D}) \leqq$

\footnotetext{
$8 \mathfrak{n}+1=\mathfrak{m}$ if $\mathfrak{m}$ is an infinite cardinal.
} 
card $(I)<$ it. We take in $\mathscr{D}$ the metric $\rho_{1}$ and consider as $T$ the identity transformation on $\mathscr{D}$. It is then a contraction. We shall form a space $\mathscr{E}=\mathscr{D} \cup(\xi)$ by adjoining to $\mathscr{D}$ a single points $\xi$ not in $\mathscr{F}$, and define the metric $\rho$ on $\mathscr{E}$ as follows :

$$
\begin{aligned}
& \rho(x, y)=\rho_{1}(x, y) \text { if } x, y \text { in } \mathscr{D}, \\
& \rho(\xi, \xi)=0, \\
& \rho(x, \xi)=\rho(\xi, x)=r^{\prime}(x) \text { for } x \text { in } \mathscr{D}, \text { where } r^{\prime}(x)=\inf r \text {, for all } r>0 \\
& \text { such that for some } i \in I, \bar{S}(x, r)>\bar{S}\left(x_{i}, r_{i}\right) .
\end{aligned}
$$

To prove that the so defined $\rho$ is a metric, we have only to prove the following inequalities :

(2) $\quad \rho_{1}(x, y)+\rho(y, \xi) \geqq \rho(x, \xi), \quad x, y$ in $\mathscr{D}$.

Proof of (1). For every $\varepsilon>0$, there exist $i, j \in I$ such that

$$
\left.\bar{S}\left(x, r^{\prime}(x)+\varepsilon\right) \supset \overline{S(} x_{i}, r_{i}\right)
$$

and

$$
\bar{S}\left(y, r^{\prime}(y)+\varepsilon\right) \supset \bar{S}\left(x_{j}, r_{j}\right) \text {. }
$$

Since

$$
\rho_{1}\left(x_{i}, x_{j}\right) \leqq r_{i}+r_{j},
$$

by the total convexity,

$$
\bar{S}\left(x_{i}, r_{i}\right) \cap \bar{S}\left(x_{j}, r_{j}\right) \neq 0 .
$$

Hence the two spheres $\bar{S}\left(x, r^{\prime}(x)+\varepsilon\right)$ and $\bar{S}\left(y, r^{\prime}(y)+\varepsilon\right)$ intersect, and

$$
\rho_{1}(x, y) \leqq r^{\prime}(x)+r^{\prime}(y)+2 \varepsilon .
$$

The first inequality is then proved because $\varepsilon$ is arbitrary.

Proof of (2). Take an arbitrary $\varepsilon>0$. There exists $i \in I$ such that $\left.\bar{S}\left(y, r^{\prime}(y)+\varepsilon\right) \supset \overline{S(} x_{i}, r_{i}\right)$. Because

$$
\bar{S}\left(x, \rho_{1}(x, y)+r^{\prime}(y)+\varepsilon\right) \supset \bar{S}\left(y, r^{\prime}(y)+\varepsilon\right) \supset \bar{S}\left(x_{i}, r_{i}\right),
$$

we have

$$
\rho(x, \xi)=r^{\prime}(x) \leqq \rho_{1}(x, y)+r^{\prime}(y)+\varepsilon .
$$

Since $\varepsilon$ is arbitrary, $\rho(x, \xi) \leqq \rho_{1}(x, y)+\rho(y, \xi)$. Clearly card $(\mathscr{E})<\mathfrak{m}+1$. 
By our hypothesis there exists an extension $\tilde{T}>T$ of $\mathscr{E}$ into $\mathscr{F}$ which is a contraction. It follows, therefore, that

$$
\rho_{1}\left(\tilde{T}(\xi), x_{i}\right)=\rho_{1}\left(\tilde{T}(\xi), \tilde{T}\left(x_{i}\right)\right) \leqq \rho\left(\xi, x_{i}\right)=r^{\prime}\left(x_{i}\right) \leqq r_{i} .
$$

Hence $\bigcap_{i \in I} \bar{S}\left(x_{i}, r_{i}\right) \neq 0$.

THEOREM 3. Let $\mathscr{F}$ be an m-hyperconvex metric space, and $T$ be a transformation of a metric space $\mathscr{D}$ into $\mathscr{F}$ with a subadditive modulus of continuity $\delta(\varepsilon)$. Let $\mathscr{E}$ be an $(\mathfrak{m}+1)$-separable space containing $\mathscr{D}$ metrically. Then there exists an extension $\tilde{T} \succ T$ to the whole of $\mathscr{E}$ with range in $\mathscr{F}$ with the same modulus of continuity $\delta(\varepsilon)$.

Proof. If we have a transfinite sequence of transformations $\left\{T_{\alpha}\right\}_{\alpha<\mu}$ such that $T_{\alpha}<T_{\beta}$ for $\alpha<\beta$ and if all these transformations have the same modulus of continuity $\delta(\varepsilon)$, and their ranges in $\mathscr{F}$, then their common extension to the union of their domains has obviously also the modulus of continuity $\delta(\varepsilon)$ and its range in $\mathscr{F}$. It follows by a classical argument that for the given transformation $T$ there exists a maximal extension $\tilde{T}>T$ with modulus of continuity $\delta(\varepsilon)$ with domain $\tilde{\mathscr{D}} \subset \mathscr{C}$ and range in $\mathscr{F}$. We shall prove that $\tilde{\mathscr{D}}$ must then necessarily be $\mathscr{E}$ and hence $\tilde{T}$ will be the required extension of our theorem.

In fact, suppose that $\tilde{\mathscr{D}}$ is a proper subset of $\mathscr{E}$. Then there exists a point $y$ in $\mathscr{E}-\tilde{\mathscr{D}}$. By our lemma, $\tilde{\mathscr{D}}$ is $\mathfrak{m}$-separable. Therefore there is in $\tilde{\mathscr{D}}$ a dense subset $\left\{p_{i}\right\}, i \in I$, card $(I)<\mathfrak{m}$. Consider in $\mathscr{F}$ the family of spheres $\left.\overline{S(T}\left(p_{i}\right), \delta\left(\rho\left(p_{i}, y\right)\right)\right)$. Clearly we have

$$
\begin{aligned}
\rho_{1}\left(\tilde{T}\left(p_{i}\right), \tilde{T}\left(p_{j}\right)\right) & \leqq \delta\left(\rho\left(p_{i}, p_{j}\right)\right) \leqq \delta\left(\rho\left(p_{i}, y\right)+\rho\left(y, p_{j}\right)\right) \\
& \leqq \delta\left(\rho\left(p_{i}, y\right)\right)+\delta\left(\rho\left(p_{j}, y\right)\right) .
\end{aligned}
$$

By m-hyperconvexity, there exists a point $z \in \mathscr{F}$ such that for every $i$ in $I$

$$
\rho_{1}\left(\tilde{T}\left(p_{i}\right), z\right) \leqq \delta\left(\rho\left(p_{i}, y\right)\right)
$$

Define a transformation $T^{\prime}$ with domain $\left\{p_{i}\right\}_{i \in I} \cup(y)$ and such that $T\left(p_{i}\right)=\tilde{T}\left(p_{i}\right)$ and $T^{\prime}(y)=z$. On the set $\left\{p_{i}\right\}_{i \in I}, T^{\prime}=\tilde{T}$, and so $T^{\prime}$ has the same modulus $\delta(\varepsilon)$. On its complete domain $T^{\prime}$ has also the modulus $\delta(\varepsilon)$ in view of (2.2). Hence it is a uniformly continuous transformation with modulus $\delta(\varepsilon)$ and domain $\left\{p_{i}\right\}_{i \in I} \cup(y)$. Therefore it has a unique extension to the closure of its domain with the same modulus of continuity. Since the closure of its domain clearly contains $\tilde{\mathscr{D}} \cup(y)$ and on $\tilde{\mathscr{D}}$ it coincides 
with $\tilde{T}$, then the extension of $T^{\prime}$ to the closure of its domain is a proper extension of $\tilde{T}$. This is a contradiction to the maximality of $\tilde{T}$. Hence our theorem is proved.

As an immediate corollary of Theorems 2 and 3 we obtain the following theorem.

THEOREM 4. Let $\mathscr{F}$ be a metric space. In order that any transformation $T$ of any metric space $\mathscr{D}$ into $\mathscr{F}$ with some subadditive modulus of continuity $\delta(\varepsilon)$ possess, for any space $\mathscr{E}$ containing $\mathscr{D}$ metrically, an extension to the whole of $\mathscr{E}$ with the same modulus $\delta(\varepsilon)$ and with range contained in $\mathscr{F}$, it is necessary and sufficient that $\mathscr{T}$ be hyperconvex.

Proof. The sufficiency follows from Theorem 3, since every metric space $\mathscr{E}$ is $(\mathfrak{m}+1)$-separable for some cardinal $\mathfrak{m}$, and $\mathscr{F}$, being hyperconvex, is m-hyperconvex.

The necessity follows from Theorem 2 , since $\mathscr{F}$ is $m$-separable for some cardinal $\mathrm{m}$, and therefore must be $\mathrm{m}$-hyperconvex if we restrict even the space $\mathscr{E}$ to be $(\mathfrak{m}+1)$-separable.

3. Properties of hyperconvex spaces; hyperconvex subsets of metric spaces. In the present section we shall compare the hyperconvexity or $\mathrm{m}$-hyperconvexity with other properties of metric spaces, and also study the class of all hyperconvex subsets of a given metric space. We introduce first the following properties of metric spaces.

Definition 1. A metric space is said to have the property $(P)$ (or $\left.\left(P_{\mathfrak{m}}\right), \mathfrak{m} \geqq 3\right)$ if for any class of closed spheres $\left.\overline{S(} x_{i}, r_{i}\right), i \in I$, where $I$ is an arbitrary class of indices (or card $(I)<\mathfrak{m}$ ), such that every couple of these spheres intersect, all the spheres intersect.

It is immediately seen that the property $(P)\left(\right.$ or $\left(P_{\mathfrak{m}}\right)$ ) is weaker than hyperconvexity (or m-hyperconvexity). In fact, if any two spheres $\bar{S}\left(x_{i}, r_{i}\right)$ and $\left.\overline{S(} x_{j}, r_{j}\right)$ intersect, then the relation $\rho\left(x_{i}, x_{j}\right) \leqq r_{i}+r_{j}$ holds.

THEOREM 1. Property $(P)$ (or $\left(P_{\mathfrak{m}}\right)$ for $\mathfrak{m}>\aleph_{0}$ ) implies the completeness of the space $\mathscr{E}$.

Proof. Take any Cauchy sequence $\left\{x_{k}\right\}$ in $\mathscr{E}$. Put $r_{k}=\sup _{l>k} \rho\left(x_{k}, x_{l}\right)$. Since $\left\{x_{k}\right\}$ is a Cauchy sequence, $r_{k}$ converges to 0 . Consider then the class of spheres $\bar{S}\left(x_{k}, r_{k}\right), k=1,2, \cdots$. It satisfies clearly the requirement of property $(P)$ (or $\left(P_{\mathfrak{m}}\right)$ for $\left.\mathfrak{m}>\aleph_{0}\right)$. Therefore there exists a point $x$ common to all $\overline{S(}\left(x_{k}, r_{k}\right)$. It follows that 


$$
\limsup _{k \rightarrow \infty} \rho\left(x, x_{k}\right) \leqq \limsup _{k \rightarrow \infty} r_{k}=0 .
$$

Hence $\lim _{k \rightarrow \infty} x_{k}=x$, which proves our theorem.

As an immediate corollary we obtain

THEOREM 1'. Hyperconvexity (or m-hyperconvexity for $\mathrm{nt}>\boldsymbol{N}_{0}$ ) $\mathrm{im}$ plies completeness of the space.

THEOREM 2. Hyperconvexity (or m-hyperconvexity) is equivalent to the property $(P)\left(\right.$ or $\left.\left(P_{\mathrm{m}}\right)\right)$ and total convexity.

Proof. In view of a previous remark it remains only to prove that the property $(P)$ (or $\left.\left(P_{\mathfrak{m}}\right)\right)$ and total convexity imply hyperconvexity (or m-hyperconvexity). But this follows immediately upon inspection of the definition or the relevant properties, and because of the fact that by total convexity if two spheres $\bar{S}\left(x_{i}, r_{i}\right)$ and $\bar{S}\left(x_{j}, r_{j}\right)$ satisfy the relation $\rho\left(x_{i}, x_{j}\right) \leqq r_{i}+r_{j}$, they must intersect.

REMARK 1. In Theorem 2, we can replace total convexity by convexity if we consider the property $(P)$ (or $\left(P_{\mathfrak{m}}\right)$ for $\left.m>\aleph_{0}\right)$. This is due to the fact that by Theorem 1 the space satisfying the property $(P)$ (or $\left.\left(P_{\mathfrak{m}}\right), \mathfrak{m}>\aleph_{0}\right)$ is already complete, and in complete spaces convexity implies total convexity. (See K. Menger [12] and N. Aronszajn [2].)

We introduce now an auxiliary notion, which will be helpful in the development of the present considerations.

DEFINITION 2. A metric space $\mathscr{E}$ is said to be almost hyperconvex (or almost $m$-hyperconvex for $m \geq 3$ ) if for any class of spheres $\bar{S}\left(x_{i}, r_{i}\right)$, $i \in I$ (or for any such class with card $(I)<\mathfrak{m}$ ), satisfying $\rho\left(x_{i}, x_{j}\right) \leqq r_{i}+r_{j}$, $i, j \in I$, and for any $\varepsilon>0$, the intersection $\left.\bigcap_{i \in I} \overline{S(} x_{i}, r_{i}+\varepsilon\right)$ is not empty.

It is clear that almost hyperconvexity (or almost m-hyperconvexity) is weaker than hyperconvexity (or $m$-hyperconvexity). For $\mathfrak{m}=3$ the notion of almost 3-hyperconvexity was first introduced under the name of almost convexity (N. Aronszajn [1]).

When we consider the different properties which we introduced for a subset of a metric space, we shall treat the subset as a space in itself with the metric induced by the metric of the space in which it lies.

TheORem 3. Consider two subsets $A$ and $B$ of a metric space $\mathscr{E}$ with equal closures in $\mathscr{E}: \bar{A}=\bar{B}$. If $A$ is almost hyperconvex (or almost m-hyperconvex), then so is $B$. 
Proof. Consider in $B$ a class of spheres $\overline{S(}\left(y_{i}, r_{i}\right), y_{i} \in B, i \in I$ ( $I$ is any class of indices or card $(I)<\mathfrak{m}$ depending on what hypothesis we accept), with $\rho\left(y_{i}, y_{j}\right) \leqq r_{i}+r_{j}$, for $i, j \in I$.

Since $B \subset \bar{A}$, there is a point $x_{i}$ in $A$ such that $\rho\left(x_{i}, y_{i}\right)<\frac{\varepsilon}{4}$ for any $\varepsilon>0$. Consider then the class of spheres $\bar{S}\left(x_{i}, r_{i}+\frac{\varepsilon}{4}\right), i \in I$. Here we have

$$
\rho\left(x_{i}, x_{j}\right) \leqq \rho\left(x_{i}, y_{i}\right)+\rho\left(y_{i}, y_{j}\right)+\rho\left(y_{j}, x_{i}\right) \leqq \frac{\varepsilon}{4}+r_{i}+r_{j}+\frac{\varepsilon}{4},
$$

which shows that $\left\{\bar{S}\left(x_{i}, r_{i}+\frac{\varepsilon}{4}\right)\right\}_{i \in I}$ satisfies the condition of almost hyperconvexity (or almost $\mathfrak{m}$-hyperconvexity). Therefore $\bigcap_{i \in I} \bar{S}\left(x_{i}, r_{i}+\frac{\varepsilon}{2}\right) \cap A$ is not empty. Let $x$ be a point in the intersection. Since $A \subset \bar{B}$, there exists a point $y$ in $B$ such that $\rho(x, y)<\frac{\varepsilon}{4}$. It follows that for $i \in I$,

$$
\rho\left(y_{i}, y\right) \leqq \rho\left(y_{i}, x_{i}\right)+\rho\left(x_{i}, x\right)+\rho(x, y) \leqq \frac{\varepsilon}{4}+r_{i}+\frac{\varepsilon}{2}+\frac{\varepsilon}{4}=r_{i}+\varepsilon
$$

Hence the proof is complete.

THEOREM 4. If a space $\mathscr{E}$ is complete and almost $(\mathfrak{m}+1)$-hyperconvex, then it is m-hyperconvex ${ }^{9}$.

Proof. Consider a class of spheres $\bar{S}\left(x_{i}, r_{i}\right), i \in I$, card $(I)<\mathfrak{m}$, with $\rho\left(x_{i}, x_{j}\right) \leqq r_{i}+r_{j}, i, j \in I$. We shall construct by induction a sequence of points $\left\{p_{k}\right\}$ in $\mathscr{E}, k=1,2, \cdots$, such that

$$
p_{k} \in \bar{S}\left(p_{k-1}, \frac{1}{2^{k-1}}+\frac{1}{2^{k}}\right) \cap \bigcap_{i \in I} \bar{S}\left(x_{i}, r_{i}+\frac{1}{2^{k}}\right), \quad k=2,3, \cdots
$$

Once the sequence $\left\{p_{k}\right\}$ is constructed, it is easy to see that it is a Cauchy sequence. In fact, $\rho\left(p_{k-1}, p_{k}\right) \leqq \frac{1}{2^{k-1}}+\frac{1}{2^{k}}$, hence $\left\{p_{k}\right\}$ is a Cauchy sequence. For its limit $p$ we have then

9 Our original theorem was as follows: if a space $\mathcal{E}$ is complete and almost $\mathfrak{m}$-hyperconvex for $\mathfrak{m} \geqq \mathfrak{S t}_{0}$, then it is $\mathfrak{m}$-hyperconvex. We are indebted to the referee for the remark, that by a slight change in the proof (which actually simplified it) one can prove the present statement. Obviously the two statements are equivalent for $\mathrm{m} \geqq \widehat{s}_{0}$, but the present one adds to our information about the case with $\mathfrak{m}<$ sto. $_{0}$. 


$$
\rho\left(x_{i}, p\right)=\lim _{k \rightarrow \infty} \rho\left(x_{i}, p_{k}\right) \leqq \lim _{k \rightarrow \infty}\left(r_{i}+\frac{1}{2^{k}}\right)=r_{i},
$$

which means that $\bigcap_{i \in I} \bar{S}\left(x_{i}, r_{i}\right) \neq 0$.

It remains to show that a sequence $\left\{p_{k}\right\}$ with property (3.1) exists. By almost $m$-hyperconvexity, there exists a point $p_{1}$ in $\bigcap_{i \in I} \bar{S}\left(x_{i}, r_{i}+\frac{1}{2}\right)$.

Suppose that the point $p_{k}$ is already defined satisfying $p_{k} \in$ $\bigcap_{i \in I} \bar{S}\left(x_{i}, r_{i}+\frac{1}{2^{k}}\right)$. If we adjoint to the spheres $\bar{S}\left(x_{i}, r_{i}\right)$ the sphere $\bar{S}\left(p_{k}, \frac{1}{2^{k}}\right)$, the condition in almost $(\mathfrak{m}+1)$-hyperconvexity is obviously satisfied and hence we can choose a point $p_{k+1}$ in the intersection

$$
\bar{S}\left(p_{k}, \frac{1}{2^{k}}+\frac{1}{2^{k+1}}\right) \cap \bigcap_{i \in I} \bar{S}\left(x_{i}, r_{i}+\frac{1}{2^{k+1}}\right) .
$$

The last theorem together with Theorem $1^{\prime}$ gives immediately

COROLlaRY 1. For $\mathfrak{m}>\boldsymbol{H}_{0}$, $\mathfrak{m}$-hyperconvexity is equivalent to almost m-hyperconvexity and completeness.

Corollary 2. Let $\mathscr{E}$ be a complete space, $\mathfrak{m} \geqq \mathfrak{S}_{0}$, and $A$ a dense subset of $\mathscr{E}$. Then the almost m-hyperconvexity of $A$ is equivalent to the m-hyperconvexity of $\mathscr{E}$.

This follows immediately from Theorems 3 and 4 .

Theorems 3 and 4 give

COROLlaRY 3. If $\mathscr{E}$ is almost $(\mathrm{m}+1)$-hyperconvex, then the completion of $\mathscr{E}$ is $\mathrm{m}$-hyperconvex.

Let $\mathscr{E}$ be an arbitrary metric space. We shall denote by $\mathscr{U}(\mathscr{E})$ the class of all nonempty closed subsets of $\mathscr{E}$. We can consider in this class the metric which was introduced by F. Hausdorff [7], and is defined as follows: for $A, B \in \mathfrak{A}(\mathscr{E})$

$$
\rho(A, B)=\max \left[\sup _{x \in A} \rho(x, B), \sup _{y \in B} \rho(y, A)\right] .
$$

The distance here may be infinite for some sets, (for example, if one of the sets $A, B$ is unbounded). Since in any case all the properties of a metric are satisfied, except that the value $+\infty$ may be taken, we shall call the space in general an extended metric space. It is easily proved that in such a space we have a natural decomposition of it into mutually disjoint metric components. Any two elements belong to the 
same component if the distance between them is finite, otherwise they belong to different components. The topology induced by the extended metric is introduced like that by a usual metric. Therefore all metric components are mutually disjoint, open and closed subsets, each of which forms a usual metric space.

Denote by $\mathfrak{A}_{\mathfrak{n}}(\mathscr{E})$ the subclass of $\mathfrak{A}(\mathscr{E})$ of all sets which are almost m-hyperconvex.

TheORem 5. $\mathfrak{U}_{\mathfrak{m}}(\mathscr{E})$ is a closed subset of $\mathfrak{U}(\mathscr{E})$ in the Hausdorff metric.

Proof. In fact, let $\left\{A_{n}\right\}$ be a sequence in $\mathfrak{U}_{\mathfrak{n}}(\mathscr{E})$, converging to $A$ in $\mathfrak{A}(\mathscr{E})$. We have to prove that $A$ is in $\mathfrak{A}_{\mathfrak{m}}(\mathscr{E})$. Consider any class of spheres $\bar{S}\left(x_{i}, r_{i}\right), i \in I$, card $(I)<\mathrm{nt}$, with $x_{i} \in A$ and $\rho\left(x_{i}, x_{j}\right) \leqq r_{i}+r_{j}$, $i, j \in I$. For any $\varepsilon>0$ consider then a positive integer $n$ such that $\rho\left(A_{n}, A\right)<\frac{\varepsilon}{4}$. Therefore for each $x_{i}$ there exists $y_{i} \in A_{n}$ such that $\rho\left(x_{i}, y_{i}\right)<_{4}^{\varepsilon}$. Clearly the class of spheres $\bar{S}\left(y_{i}, r_{i}+\frac{\varepsilon}{4}\right)$ satisfies the condition of almost m-hyperconvexity in $A_{n}$. Hence if we add $\frac{\varepsilon}{4}$ to all radii, there exists a point $y$ in $A_{n} \cap \bigcap_{i \in I} \bar{S}\left(y_{i}, r_{i}+\frac{\varepsilon}{2}\right)$. Since $\rho\left(A_{n}, A\right)<\frac{\varepsilon}{4}$, there is a point $x$ in $A$ such that $\rho(x, y)<\frac{\varepsilon}{4}$. That $x$ belongs to $\bigcap_{i \in I} \bar{S}\left(x_{i}, r_{i}+\varepsilon\right)$ follows from the inequalities:

$$
\rho\left(x, x_{i}\right) \leqq \rho(x, y)+\rho\left(y, y_{i}\right)+\rho\left(y_{i}, x_{i}\right) \leqq{ }_{4}^{\varepsilon}+r_{i}+\frac{\varepsilon}{2}+\frac{\varepsilon}{4}=r_{i}+\varepsilon
$$

Hence the theorem is proved.

REMARK 2. If $\mathscr{E}$ is complete and $m \geqq \boldsymbol{w}_{0}$, then clearly all sets in $\mathfrak{U}(\mathcal{E})$ are complete as metric spaces in themselves, and hence all sets in $\mathfrak{A}_{\mathfrak{m}}(\mathfrak{Z})$ are $\mathfrak{m}$-hyperconvex by Theorem 4.

Even if the space $\mathscr{E}$ is not hyperconvex (or $m$-hyperconvex) itself, it may contain a large class of hyperconvex subsets, for example, all isometric images of segments of the real line. If itself is hyperconvex, then it contains always a large class of hyperconvex subsets, which even satisfy a stronger property, which we shall call the external hyperconvexity relative to $c$, and which is defined as follows: 
DeFINITION 3. A set $A$ contained in $\mathscr{E}$ is externally hyperconvex, in short ext. hyperconvex, relative to $\mathscr{E}$ if for any class of spheres $\bar{S}\left(x_{i}, r_{i}\right)$ in $\mathscr{E}\left(x_{i}\right.$ not necessarily in $\left.A\right)$ satisfying $\rho\left(x_{i}, x_{j}\right) \leqq r_{i}+r_{j}$ and $\rho\left(x_{i}, A\right) \leqq r_{i}$ for $i, j \in I, A \cap \bigcap_{i \in I} \bar{S}\left(x_{i}, r_{i}\right)$ is not empty.

Similar definitions can be given for ext. m-hyperconvexity. The following theorem with some changes will also be valid for the case of ext. m-hyperconvexity.

THEOREM 6. Any nonempty intersection of a class of spheres, $\bigcap_{k \in K} \bar{S}\left(a_{k}, \rho_{k}\right), K$ being an arbitrary class of indices, of a hyperconvex space $\mathscr{E}$ is externally hyperconvex relative to $\mathscr{E}$.

Proof. Let

$$
A=\bigcap_{k \in K} \bar{S}\left(\alpha_{k}, \rho_{k}\right) \neq 0 .
$$

Consider an arbitrary class of spheres $\left\{\bar{S}\left(x_{i}, r_{i}\right)\right\}_{i \in I}$ with $\rho\left(x_{i}, x_{j}\right) \leqq r_{i}+r_{j}$ and $\rho\left(x_{i}, A\right) \leqq r_{i}$ for $i, j \in I$. The class of all spheres $\bar{S}\left(a_{k}, \rho_{k}\right)$ and $\bar{S}\left(x_{i}, r_{i}\right)$ satisfies the requirements of hyperconvexity in $\mathscr{E}$. For if $k, l \in K$, we have clearly $\rho\left(a_{k}, a_{l}\right) \leqq \rho_{k}+\rho_{l}$ by hypothesis. The case where $i, j \in I$ is obvious. Finally for $k \in K, i \in I$, we have, for $\varepsilon>0$ and some point $y_{i} \in A, \rho\left(x_{i}, y_{i}\right)<r_{i}+\varepsilon$ (since $\left.\rho\left(x_{i}, A\right) \leqq r_{i}\right)$, and therefore

$$
\rho\left(a_{k}, x_{i}\right) \leqq \rho\left(a_{k}, y_{i}\right)+\rho\left(x_{i}, y_{i}\right) \leqq \rho_{k}+r_{i}+\varepsilon \text {. }
$$

Since $\varepsilon$ is arbitrary, $\rho\left(a_{k}, x_{i}\right) \leqq \rho_{k}+r_{i}$. Therefore there is a point in the intersection

$$
\bigcap_{i \in I} \bar{S}\left(x_{i}, r_{i}\right) \bigcap_{k \in K} \bar{S}\left(a_{k}, \rho_{k}\right)=A \cap \bigcap_{i \in I} \bar{S}\left(x_{i}, r_{i}\right)
$$

There are several interesting properties of ext. hyperconvex subsets of $\mathscr{E}$. We shall mention here only one, and that is the following.

THEOREM 7. If $A$ is ext. hyperconvex relatively to $\mathscr{E}$, then for every point $x$ in $\mathscr{E}$ there exists a point $a$ in $A$ realizing the minimum distance from $x$ to $A$.

Proof. Consider for $r=\rho(x, A)$, the sphere $\bar{S}(x, r)$. Clearly it satisfies the requirements of ext. hyperconvexity. Therefore $A \cap \bar{S}(x, r) \neq 0$. Let $a$ be a point in the intersection. It follows that $a$ is on the boundary of $\bar{S}(x, r)$ and $\rho(\alpha, x)=\rho(x, A)$.

We shall pass now to purely topological properties in order to show 
that hyperconvex sets form a very restricted class from a topological point of view. We shall recall that a subset $A$ of $\mathscr{E}$ is called a retract of $\mathscr{E}$ if there is a continuous transformation $T$, called retraction, of $\mathscr{E}$ onto $A$ such that $T(x)=x$ for $x$ in $A$. A metrizable space $A$ is called an absolute retract (See $\mathrm{K}$. Borsuk [5]) if for any metrizable space $\mathscr{E}$, in which $A$ is topologically embedded, $A$ is a retract of $\mathscr{E}$. Absolute retracts are compact, connected, locally connected, and all their homology groups and homotopy groups are the same as those of a single point. A metrizable space $A$ will be called a generalized absolute retract if for every metrizable space $\mathscr{E}$, in which $A$ is topologically embedded and closed, $A$ is a retract of $\mathscr{E}$. The generalized absolute retracts have many properties similar to those of the absolute retracts. However, a generalized absolute retract is not always compact; instead of compactness it has the property of being an absolute $G_{\delta}{ }^{10}$. A generalized absolute retract is an absolute retract if and only if it is compact.

If $T$ is a retraction of a metric space $\mathscr{E}$ onto its subset $A$, we shall call it contracting retraction if it is also a contraction.

THeOREM 8. If $A$ is hyperconvex, and is contained metrically in a space $\mathscr{E}$, then there exists a contracting retraction on $\mathscr{E}$ onto $A$.

Proof. In fact, consider the identity as a transformation of $A$ onto $A$. By the extension property of a hyperconvex space, Theorem $4, \S 2$, we can extend this transformation with conservation of modulus $\delta(\varepsilon)=\varepsilon$ to the whole of $\mathscr{E}$ and with range in $A$; the extension is clearly a contracting retraction of $\mathscr{E}$ onto $A$.

COROLlaRY 4. If $A$ is hyperconvex, then it is a generalized absolute retract.

Proof. In fact, if $A$ is contained topologically in $\mathscr{E}$ and is closed in ' $\delta$ ', then by a theorem of F. Hausdorff (F. Hausdorff [8]), we can change the metric of $\mathscr{E}$ into a topologically equivalent metric, which coincides with the hyperconvex metric of $A$ on $A$. We then apply the preceding theorem and obtain a retraction of $\mathscr{E}$ onto $A$.

Corollary 5. If $A$ is hyperconvex and compact, it is an absolute retract.

Proof. This follows immediately from Corollary 4 and the definition.

10 This means that it can be metrized with a complete metric. 
THEOREM 9. If $\mathscr{E}$ is a hyperconvex space, then a subset $A$ of $\mathscr{E}$ is hyperconvex if and only if it is a retract of $\mathscr{E}$ by a contracting retraction.

Proof. If $A$ is hyperconvex, then the existence of a contracting retraction is given by Theorem 8 .

Conversely, if $T$ is a contracting retraction of $\mathscr{O}$ onto $A$, consider any class of spheres $\bar{S}\left(x_{i}, r_{i}\right), x_{i} \in A$ and $\rho\left(x_{i}, x_{j}\right) \leqq r_{i}+r_{j}$ for $i, j \in I$. Because $\mathscr{E}$ is hyperconvex, there exists a point $p$ in $\bigcap_{i \in I} \bar{S}\left(x_{i}, r_{i}\right)$. Then it follows that

$$
\rho\left(T(p), x_{i}\right)=\rho\left(T(p), T\left(x_{i}\right)\right) \leqq \rho\left(p, x_{i}\right) \leqq r_{i} .
$$

Hence $T(p) \in \bigcap_{i \in I} A \cap \bar{S}\left(x_{i}, r_{i}\right)$.

4. Hyperconvex real Banach spaces. Hyperconvex real Banach spaces were recently considered by L. Nachbin [14]. He introduced them by using the property $(P)$ of $\S 3$, which clearly is sufficient since linear normed spaces are always totally convex. His results are of two kinds. First, he considers the extension of a linear transformation $T$ from a real Banach space $\mathscr{E}$ into a real Banach space $\mathscr{F}$ and proves that the hyperconvexity of $\mathscr{F}$ is necessary and sufficient for the possibility of extending $T$ to a linear transformation $\tilde{T}$ of the whole of $\mathscr{E}$ into $\mathscr{F}$, with conservation of bound, $\|T\|=\|\tilde{T}\|$, for any $\mathscr{E}$ and $T$. This result was obtained by N. Aronszajn in 1929, but was never published $^{11}$. The second kind of Nachbin's results concerns the characterization of hyperconvex real Banach spaces as spaces $\mathscr{C}(\mathscr{C})$ of all real continuous functions on extremally disconnected compact Hausdorff spaces $\mathscr{K}$. This interesting result was obtained by Nachbin under the assumption that the closed unit sphere in a hyperconvex real Banach space possesses an extreme point. J. L. Kelley [11] proved this result of Nachbin without assuming the existence of extreme points of the unit sphere. He used a characterization of hyperconvex Banach spaces introduced by D. B. Goodner $[6]^{12}$, and constructed explicitly the space $\mathscr{H}$ by using the extreme points of the unit sphere in the conjugate space. Kelley's proof clearly settles in the affirmative Nachbin's conjecture that the unit sphere in a hyperconvex real Banach space always has an extreme point. We are going to prove here in a more direct

11 This result is not exactly a special case of our general theorem from $\$ 2$, since it adds to the hypothesis the linearity of $T$ and to the thesis the linearity of the extension $\tilde{T}$. But this additional point can be arranged in a similar way as in the classical proof of the Hahn-Banach theorem, and we shall not give here the arrangement since it was done by Nachbin. We shall remark, however, that by using the idea of our proof of necessity in Theorem 2, $\S 2$ (introduction of $r^{\prime}(x)$ ) Nachbin's proof of necessity could be simplified.

12 This characterization is akin to our Theorem 8 , 3 . 
way Nachbin's conjecture without going out of the original Banach space, and we shall use only its hyperconvexity and some general theorems about convex sets.

We start by introducing the general notions and properties concerning abstract convex sets $^{13}$, which we shall need ${ }^{14}$. For the sake of completeness we introduce also some standard notations. In an abstract real vector space $\mathscr{Y}, x, y \in \mathscr{Y}, x \neq y, \alpha$ and $\beta$ reals, we call

$$
\overleftrightarrow{x y}=\stackrel{\leftrightarrow}{y x}=\underset{w}{\mathrm{E}}[w=\alpha x+\beta y, \alpha+\beta=1]
$$

the straight line through $x$ and $y$,

$$
\stackrel{\leftrightarrow}{x y}=\overleftrightarrow{y x}=\underset{w}{\mathrm{E}}[w=\alpha x+\beta y, \beta \geqq 0, \alpha+\beta=1]
$$

the half line from $x$ through $y$,

$$
[x ; y]=[y ; x]=\underset{w}{\mathrm{E}}[w=\alpha x+\beta y, \alpha \geqq 0, \beta \geqq 0, \alpha+\beta=1]
$$

the segment joining $x$ and $y$,

$$
(x ; y]=[y ; x)=\underset{w}{\mathrm{E}}[w=\alpha x+\beta y, \alpha \geqq 0, \beta>0, \alpha+\beta=1]
$$

the half open segment from $y$ to $x$,

$$
(x ; y)=(y ; x)=\mathrm{E}_{w}[w=\alpha x+\beta y, \alpha>0, \beta>0, \alpha+\beta=1]
$$

the open segment joining $x$ and $y$.

If $x=y$, we extend the definitions of segments in the following way:

$$
[x ; y]=[x ; y)=(y ; x]=(x)=(y),
$$

and

$$
(x ; y)=0 \text {. }
$$

A subset $V^{\prime}$ of $\mathscr{V}^{\prime}$ is called a linear variety if with any two points $x, y x \neq y$, it contains the whole straight line $\overleftrightarrow{x y}$. Every linear subspace is a linear variety. Every linear variety is obtained from one and only one linear subspace by translation. The dimension of the linear variety is the same as that of the linear subspace. In the case of finite dimension, it has therefore the natural topology of finite dimensional vector spaces.

A set $K$ contained in $\mathscr{V}$ is convex if with any two points $x, y$ it

13 Convexity means here the usual convexity in vector spaces as distinguished from metrical convexity; the latter means that the set is provided with a convex metric.

${ }_{14}$ These general notions about convex sets were introduced by N. Aronszajn. 
contains the whole segment $[x ; y]$. A convex set $K$ is linearly closed if its intersection with any finite dimensional linear variety is closed in the natural topology of the linear variety. It is well known that $K$ is linearly closed if and only if its intersection with any straight line is closed on the straight line.

Let $K \subset \mathscr{Y}$ be a convex set. There exists the smallest linear variety containing it, that is, the linear variety generated by $K$. Every linear variety is linearly closed. For every convex set $K$ there exists the smallest linearly closed convex set $\tilde{K}$ containing $K . \quad \tilde{K}$ is called the linear closure of $K$.

A point $x$ in $K$ is an inner point of $K$ if and only if for every $y$ in $K, y \neq x, \overleftrightarrow{x y} \cap K$ contains $x$ in its interior (relative to $\overleftrightarrow{x y}$ ).

A point $x$ in $K$ is a border point of $K$ if and only if for some $y$ in

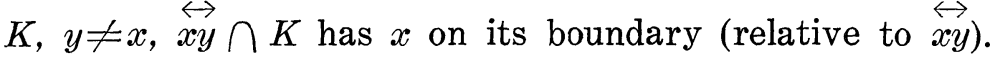

A point $x$ in $K$ is an extreme point of $K$ if and only if for every $y$ in $K, y \neq x, \overleftrightarrow{x y} \cap K$ has $x$ on its boundary (relative to $\overleftrightarrow{x y}$ ).

For any convex set $K$ containing more than one point the extreme points are at the same time border points, and the set of inner points and the set of border points form a disjoint decomposition of $K$. When $K$ is reduced to a single point $x$, the usual logical interpretation of our definitions would be that $x$ is an inner point and also an extreme point, without being a border point. We shall agree, however, in this special case still to consider $x$ as a border point. This is the only case in which an inner point is also an extreme point or a border point. It is well known that for any convex set $K$ the set of its inner points is convex, but it may be empty.

Lemma 1. Let $x$ in $K$ be an inner point of $K$. Then the linear closure $\tilde{K}$ of $K$ can be obtained as follows:

$$
\tilde{K}=\underset{y \in \subset)}{\mathrm{E}}[x ; y) \subset K .
$$

Proof. Denote the right-hand side of the equation by $K_{1}$. Clearly $K$ is contained in $K_{1}$. For any $y$ in $K_{1}, y$ belongs to the closure of $[x ; y)$, and therefore is in $\tilde{K}$.

On the other hand, $K_{1}$ is convex and linearly closed. In fact, for any $y, z$ in $K_{1}$ consider $\alpha y+\beta z, \alpha \geqq 0, \beta \geqq 0, \alpha+\beta=1$. Since $[x ; \alpha y+$ $\beta z)=\underset{w}{\mathrm{E}}[w=\gamma x+\delta(\alpha y+\beta z), \gamma>0, \delta \geqq 0, \gamma+\delta=1]$, and $\gamma x+\delta(\alpha y+\beta z)=$ $\alpha(\gamma x+\delta y)+\beta(\gamma x+\delta z)$ belongs to $K$ for all $\alpha, \beta, \gamma$, and $\delta$ as prescribed above, then $\alpha y+\beta z$ belongs to $K_{1}$ for all $\alpha \geqq 0, \beta \geqq 0, \alpha+\beta=1$. To prove that $K_{1}$ is linearly closed, we have to show that if $(y ; z) \subset K_{1}$, then $y$ 
(or $z$ ) belongs to $K_{1}$. Since $(y ; z)=\underset{w}{\mathrm{E}}[w=\alpha y+\beta z, \alpha>0, \beta>0, \alpha+\beta=1]$, then $\underset{v}{\mathrm{E}}[v=\gamma x+\delta(\alpha y+\beta z), \gamma>0, \delta \geqq 0, \gamma+\delta=1]$ is contained in $\mathrm{K}$. Because $x$ is an inner point of $K$, we have for some $\alpha_{0}>0, \beta_{0}>0, \alpha_{0}+\beta_{0}=1$ and some $\varepsilon>0$,

$$
(1+\varepsilon) x-\varepsilon\left(\alpha_{0} y+\beta_{0} z\right) \text { belongs to } K .
$$

To prove $[x ; y) \subset K$, we have to prove that $\mu x+\nu y$ belongs to $K$ for $\mu>0, \nu \geqq 0, \mu+\nu=1$. To this effect consider the following convex combination:

$$
\begin{array}{r}
\frac{\varepsilon \beta_{0}}{\varepsilon \beta_{0}+\delta \beta}(\gamma x+\delta(\alpha y+\beta z))+\frac{\delta \beta}{\varepsilon \beta_{0}+\delta \beta}\left((1+\varepsilon) x-\varepsilon\left(\alpha_{0} y+\beta_{0} z\right)\right) \\
=\frac{\varepsilon \beta_{0} \gamma+(1+\varepsilon) \delta \beta}{\varepsilon \beta_{0}+\delta \beta} x+\frac{\varepsilon \beta_{0} \delta \alpha-\varepsilon \alpha_{0} \delta \beta}{\varepsilon \beta_{0}+\delta \beta} y .
\end{array}
$$

It is easy to check that for every $0<\mu \leqq 1$ we can choose $\beta$ and $\delta$ with $0<\beta<1$ and $0 \leqq \delta<1$ such that $\frac{\varepsilon \beta_{0} \gamma+(1+\varepsilon) \delta \beta}{\varepsilon \beta_{0}+\delta \beta}=\mu$, which completes the proof.

Let $K$ be a convex set. We introduce for points $x, y$ in $K$ an equivalence relation $x \sim y$ as follows:

if $x=y$ then $x \sim y$;

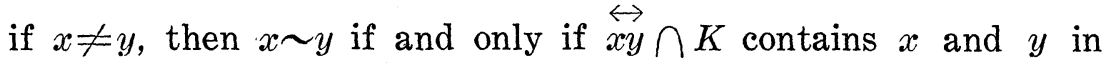
its interior (relative to $\leftrightarrow x y$ ).

It is easy to prove that the relation $x \sim y$ is actually an equivalence relation. Hence $K$ is decomposed into mutually disjoint equivalence classes. Any of these equivalence classes will be called a cell of $K$. Then to each $x$ in $K$ there exists one and only one cell $C_{x}$ such that $x \in C_{x}$.

We shall list now a series of properties of cells of a linearly closed convex set $K$; their proofs are easy and will be omitted here.

A cell $C$ is a convex set and every point of $C$ is an inner point of $C$. The linear closure $\tilde{C}$ of a cell $C$ is a union of cells, and can be determined by the formula of Lemma 1 . The inner points of $\tilde{C}$ are the points of $C . \tilde{C}$ always has border points and therefore border cells, unless $C=\tilde{C}$. This last case presents itself only if $C$ is a linear variety; that includes also the case when $C$ is reduced to a single point $x$ (variety of dimension 0 ). The single point $x$ is then an extreme point of $K$. If $x$ is a border point of $\tilde{C}$, then $\tilde{C}_{x}$ is composed of border points of $\tilde{C}$. For 
any cell $C$ an extreme point of $\tilde{C}$ is at the same time an extreme point of $K$.

As usual we shall denote, for $A \subset \mathscr{Y}$ and $\alpha$ real, by $\alpha A$ the set of all elements of the form $\alpha x, x \in A$. For any two subsets $A$ and $B$ of $\mathscr{Y}, A+B$ denotes the set of all elements representable in the form $x+y, x \in A, y \in B$. These notations will also be used when one of the sets is reduced to a single element.

THEOREM 1. Let $x$ be an element of a linearly closed convex subset $K$ of a vector space $\mathscr{Y}$. Then for every $\alpha>0$

$$
K \cap[-\alpha K \dot{ }(1+\alpha) x]=\tilde{C}_{x} \cap\left[-\alpha \tilde{C}_{x} \dot{+}(1+\alpha) x\right] .
$$

Proof. That the left-hand side of (4.1) contains the right-hand side is obvious. To prove the converse take an element $y$ in $K \cap[-\alpha K+$ $(1+\alpha) x]$. It follows that $y$ belongs to $K$ and there exists $z$ in $K$ such that $y=-\alpha z+(1+\alpha) x$. This gives $x=\frac{1}{1+\alpha} y+\frac{\alpha}{1+\alpha} z$. Since $\alpha>0, x$ belongs to $(y ; z)$. Therefore the whole open interval $(y ; z)$ is contained in $C_{x}$. Hence $[x ; y) \subset C_{x}$, and by Lemma $1, y$ is in $\tilde{C}_{x}$. Similarly, $z$ is in $\tilde{C}_{x}$. Therefore $y=-\alpha z+(1+\alpha) x$ belongs to $\tilde{C}_{x} \cap\left[-\alpha \tilde{C}_{x} \dot{+}(1+\alpha) x\right]$.

REMARK. If in the vector space $\mathscr{V}$ we have a topology agreeing with the linear structure of $\mathscr{Y}$ (for example, locally convex or normed topology), then a convex set closed in this topology is, à fortiori, linearly closed. But the converse is not necessarily true. Even if $K$ is closed in the topology, the linear closure $\tilde{C}$ of a cell $C$ of $K$ may not be closed in the topology. In view of this fact the result of Theorem 1 may seem somehow surprising, since it shows that if $K$ is closed but $\tilde{C}_{x}$ is not closed, then still $\tilde{C}_{x} \cap\left[-\alpha \tilde{C}_{x} \dot{+}(1+\alpha) x\right]$ is closed.

LEMMA 2. If $\bar{S}$ is the closed unit sphere of a normed vector space $\mathscr{Y}$, then for any $x$ in $\mathscr{P}$ and $\alpha>0$,

$$
-\alpha \bar{S}+(1+\alpha) x=\overline{S(}(1+\alpha) x, \alpha) .
$$

In fact, if $y$ is an element in $\mathscr{V}$ such that $\|y-(1+\alpha) x\| \leqq \alpha$, then there exists an element $z$ in $\bar{S}$ satisfying $-\alpha z=y-(1+\alpha) x$. Therefore $y=-\alpha z+(1+\alpha) x$. The converse is also true by reversing the above argument.

Let $\mathscr{B}$ be a hyperconvex real Banach space, the metric being given by the norm, $\rho(x, y)=\|x-y\|$. To prove Nachbin's conjecture that the 
closed unit sphere $\bar{S}$ possesses an extreme point, we shall prove first the following lemma.

Lemma 3. Let $x \in \bar{S}$ and $\alpha>1$. Then there exists a border point $y$ of $\tilde{C}_{x}$ such that

$$
\tilde{C}_{y} \subset \tilde{C}_{x} \cap\left[-\alpha \tilde{C}_{x} \dot{+}(1+\alpha) x\right]
$$

Proof. Consider first the case when

$$
\tilde{C}_{x}=\tilde{C}_{x} \cap\left[-\alpha \tilde{C}_{+}(1+\alpha) x\right] .
$$

Then we can take for $y$ any border point of $\tilde{C}_{x}$. Such a point exists since $\tilde{C}_{x}$ is contained in $\bar{S}$, and hence cannot be a linear variety, unless it is of dimension 0 , that is, a single point. But then the same single point is a border point of $\tilde{C}_{x}$.

Now consider the case when $\tilde{C}_{x} \neq \tilde{C}_{x} \cap\left[-\alpha \tilde{C}_{x} \dot{+}(1+\alpha) x\right]$. We put

$$
A=\bar{S} \cap \bar{S}\left(x, \frac{2}{1+\alpha}\right) \text {. }
$$

For every $z$ in $A$ consider the spheres

$$
\bar{S}\left(\frac{\alpha+1}{\alpha-1} z, \frac{2}{\alpha-1}\right) \text {. }
$$

The class of all these spheres together with $\bar{S}=\bar{S}(0,1)$ satisfies the requirements in the condition of hyperconvexity, since for any $z, z^{\prime}$ in $A$ we have

$$
\left\|\frac{\alpha+1}{\alpha-1} z^{\prime}-\frac{\alpha+1}{\alpha-1} z\right\|=\frac{\alpha+1}{\alpha-1}\left\|z^{\prime}-z\right\| \leqq \frac{\alpha+1}{\alpha-1}\left(\left\|z^{\prime}-x\right\|+\|x-z\|\right) \leqq 2 \cdot \frac{2}{\alpha-1},
$$

and

$$
\left\|\frac{\alpha+1}{\alpha-1} z-0\right\| \leqq \frac{\alpha+1}{\alpha-1}=\frac{2}{\alpha-1}+1
$$

Therefore there exists a point $y$ such that

$$
y \in \bar{S} \cap \bigcap_{z \in A} \bar{S}\left(\frac{\alpha+1}{\alpha-1} z, \frac{2}{\alpha-1}\right) .
$$

We shall prove that $\tilde{C}_{y} \subset \tilde{C}_{x} \cap\left[-\alpha \tilde{C}_{x}+(1+\alpha) x\right]$. Since $x$ belongs to $A$, then by applying Lemma 2 and Theorem 1 , we have 


$$
\begin{aligned}
y \in \bar{S} \cap \bar{S}\left(\begin{array}{cc}
\alpha+1 \\
\alpha-1
\end{array}, \frac{2}{\alpha-1}\right) & =\bar{S} \cap\left[-\begin{array}{c}
2 \\
\alpha-1 \\
\bar{S}+\frac{\alpha+1}{\alpha-1} x
\end{array}\right] \\
& =\tilde{C}_{x} \cap\left[-\frac{2}{\alpha-1} \tilde{C}_{x}+\frac{\alpha+1}{\alpha-1} x\right] .
\end{aligned}
$$

It follows that $y$ belongs to $\tilde{C}_{x}$ and $\tilde{C}_{y} \subset \tilde{C}_{x}$. We still have to show that $\tilde{C}_{y} \subset\left[-\alpha \tilde{C}_{x}+(1+\alpha) x\right]$. Suppose this is not true. Then there exists an element $u$ such that

$$
u \in \tilde{C_{y}}-\left[-\alpha \tilde{C_{x}}+(1+\alpha) x\right] .
$$

Since $u$ belongs to $\tilde{C}_{y} \subset \tilde{C}_{x}, u$ is in $\tilde{C}_{x}$, and since $x \in\left[-\alpha \tilde{C}_{x} \dot{+}(1+\alpha) x\right]$, we have $u \neq x$. Consider the straight line $\overleftrightarrow{u x}$ and its intersection with $\bar{S}$. Because $\bar{S}$ is linearly closed and bounded, there exist $u^{\prime}$ and $x^{\prime}$ in $\bar{S}$ such that $\stackrel{\leftrightarrow}{u x} \cap \bar{S}=\left[u^{\prime} ; x^{\prime}\right]$. We can choose the notations so that $u$ belongs to $\left[u^{\prime} ; x\right]$, and $x$ belongs to $\left[u ; x^{\prime}\right] ; u$ may be equal to $u^{\prime}$, but $x$ must be different from $x^{\prime}$. In fact, by Lemma 1 , since $u \in \tilde{C}_{y} \subset \tilde{C}_{x},(u ; x] \subset C_{x}$. Therefore $x$ and any point in $(u ; x]$ are equivalent, and $x$ must be in the interior of $\left[u^{\prime} ; x^{\prime}\right]$. This implies that $x \neq x^{\prime}$. It follows that all points in $\left(u^{\prime} ; x^{\prime}\right)$ belong to $C_{x}$. Hence $x^{\prime}$ belongs to $\tilde{C}_{x}-C_{x}$. Introduce the point $v$ by equation $u=-\alpha v+(\alpha+1) x$. Hence, by (4.4), $v \notin \tilde{C}_{x}$ and

$$
v=-\frac{1}{\alpha} u+\frac{\alpha+1}{\alpha} x, \quad x=\frac{1}{\alpha+1} u+\frac{\alpha}{\alpha+1} v .
$$

It follows that $x \in(u ; v) \subset \overleftrightarrow{u_{x}}$. Since $\left[u ; x^{\prime}\right] \subset \stackrel{\leftrightarrow}{u x},\left[u ; x^{\prime}\right] \subset \tilde{C}_{x}$ and $v \notin \tilde{C}_{x}$, we have $x^{\prime} \in(u ; v)$. Therefore by $(4.5)$, we have $\left\|x^{\prime}-u\right\|<\|v-u\|=$ $\frac{1+\alpha}{\alpha}\|x-u\|$, and $\|x-u\|>\frac{\alpha}{1+\alpha}\left\|x^{\prime}-u\right\|$. It follows that

$$
\begin{aligned}
\left\|x^{\prime}-x\right\| & =\left\|x^{\prime}-u\right\|-\|x-u\| \\
& <\left(1-\frac{\alpha}{1+\alpha}\right)\left\|x^{\prime}-u\right\| \leqq \frac{1}{1+\alpha}\left(\left\|x^{\prime}\right\|+\|u\|\right) \leqq \frac{2}{1+\alpha} .
\end{aligned}
$$

This shows that $x^{\prime} \in A$. Following (4.3),

$$
y \in \bar{S} \cap\left[-\frac{2}{\alpha-1} \bar{S}+\frac{\alpha+1}{\alpha-1} x^{\prime}\right]=\tilde{C}_{x^{\prime}} \cap\left[-\frac{2}{\alpha-1} \tilde{C}_{x^{\prime}} \dot{+} \frac{\alpha+1}{\alpha-1} x^{\prime}\right] .
$$

Therefore $y$ belongs to $\tilde{C}_{x^{\prime}}$. Hence $u \in \tilde{C}_{y} \subset \tilde{C}_{x^{\prime}}$ and $x \in\left[u ; x^{\prime}\right] \subset \tilde{C}_{x^{\prime}}$, which is impossible because it was shown above that $x^{\prime} \in \tilde{C}_{x}-C_{x}$ and therefore $\tilde{C}_{x^{\prime}} \subset \tilde{C}_{x}-C_{x}$. Consequently $\tilde{C}_{y} \subset\left[-\alpha \tilde{C}_{x}+(1+\alpha) x\right]$. 
To show that $y$ is a border point of $\tilde{C}_{x}$ we observe that otherwise $\tilde{C}_{y}=\tilde{C}_{x}$, and so $\tilde{C}_{x}=\tilde{C}_{x} \cap\left[-\alpha \tilde{C}_{x}+(1+\alpha) x\right]$, against our assumption.

Hence the lemma is proved.

THEOREM 2. Let $\bar{S}$ be the closed unit sphere of a hyperconvex real Banach space $\mathscr{B}$. For any point $x$ in $\bar{S}, \tilde{C}_{x}$ contains an extreme point of $\bar{S}$.

Proof. For $y, z$ in $\bar{S}$ define a partial order relation, $y<z$, as follows :

if $y=z$, then $y<z$,

if $y \neq z$, then $y<z$ if and only if $y$ is a border ponit of $\tilde{C}_{z}$ and

$$
\tilde{C}_{y} \subset \tilde{C}_{z} \cap\left[-2 \tilde{C}_{z}+3 z\right] \text {. }
$$

It is immediately verified that the relation $\prec$ is in fact a partial order relation.

Consider now an ordered subset $A$ of $\overline{S .}$ We claim that there exists a point $y$ in $\bar{S}$ such that $y<z$ for all $z$ in $A$. We notice first that $\tilde{C}_{z} \cap\left[-2 \tilde{C}_{z}+3 z\right]=\bar{S} \cap \bar{S}(3 z, 2)$. Consider then the class of all spheres $\bar{S}=\bar{S}(0,1)$ and $\bar{S}(3 z, 2)$ for $z \in A$. For any $z$ and $z^{\prime} \neq z$ in $A$ we can suppose that $z \prec z^{\prime}$ ( $A$ being ordered) and hence

$$
\begin{aligned}
z \in \bar{S} \cap \bar{S}(3 z, 2) & =\tilde{C}_{z} \cap\left[-2 \tilde{C}_{z}+3 z\right] \subset \tilde{C}_{z} \subset \tilde{C}_{z^{\prime}} \cap\left[-2 \tilde{C}_{z^{\prime}}+3 z^{\prime}\right] \\
& =\bar{S} \cap \bar{S}\left(3 z^{\prime}, 2\right) .
\end{aligned}
$$

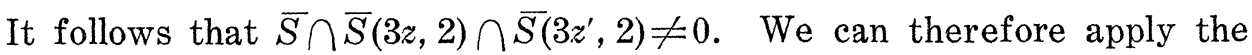
hyperconvexity to this class of spheres and find a point

$$
y_{0} \in \bigcap_{z \in A}\{\bar{S} \cap \bar{S}(3 z, 2)\}=\bigcap_{z \in A}\left\{\widetilde{C_{z}} \cap\left[-2 \tilde{C_{z}}+3 z\right]\right\} .
$$

If the ordered set $A$ has a smallest point we can take it as $y$. If, however, there is no smallest point in $A$, for each $z \in A$ there exists $z^{\prime} \in A$ with $z^{\prime} \prec z$. Then $y_{0} \in \tilde{C}_{z^{\prime}}$. Hence $y_{0}$ is together with $z^{\prime}$ a border point of $\tilde{C}_{z}$ and $\tilde{C}_{y_{0}} \subset \tilde{C}_{z}, \subset \tilde{C}_{z} \cap\left[-2 \tilde{C}_{z}+3 z\right]$. Thus $y_{0} \prec z$ and we can put $y=y_{0}$.

By a classical argument it follows now that there exists a minimal element $x_{0}$ in $\bar{S}$ which is smaller than an arbitrarily chosen element $x$ in $\bar{S}$. We are going to prove that $x_{0}$ is an extreme point of $\overline{S .}$ In fact, by Lemma 3 , there exists a point $y$, which is a border point of $\tilde{C}_{x_{0}}$ and satisfies $\tilde{C}_{y} \subset \tilde{C}_{x_{0}} \cap\left[-2 \tilde{C}_{x_{0}}+3 x_{c}\right]$. This means that $y<x_{0}$. Since $x_{0}$ is 
minimal, we have $y=x_{0}$. But $y$ is a border point of $\tilde{C_{x_{3}}}$, and $x_{0}$ is an inner point of $\widetilde{C}_{x_{0}} ; y=x_{0}$ can happen only if $\tilde{C_{x_{0}}}$ is reduced to a single point $x_{0}$. Hence $x_{0}$ is an extreme point of $\bar{S}$, contained in $\widetilde{C}_{x}$.

5. The spaces $\mathscr{C}(\mathscr{H})$ for compact Hausdorff spaces $\mathscr{C}$. Nachbin's theorem shows that the space $\mathscr{C}(\mathscr{C})$ of all continuous real functions on an extremally disconnected compact Hausdorff space $\mathscr{H}$ with the usual norm, $\|\varphi\|=\sup [|\varphi(x)|, x \in \mathscr{C}]$ is hyperconvex, and every hyperconvex real Banach space is a space $\mathscr{C}(\mathscr{C})$ for some extremally disconnected compact Hausdorff space $\mathscr{H}$ (Theorem of Nachbin-Kelley). This suggests an investigation from the point of view of m-hyperconvexity of the spaces $\mathscr{C}(\mathscr{H})$ for general compact Hausdorff spaces $\mathscr{H}$. We prove first

\section{THEOREM 1. Every space $\mathscr{C}(\mathscr{H})$ is $\widehat{H}_{0}$-hyperconvex ${ }^{15}$.}

Proof. Suppose that we have a finite number of spheres $\bar{S}\left(\varphi_{k}, r_{k}\right)$, $k=1,2, \cdots, n$, such that $\left\|\varphi_{k}-\varphi_{l}\right\| \leqq r_{k}+r_{l}$ for $1 \leqq k, l \leqq n$. By definition of the norm in $\mathscr{C}(\mathscr{H})$ we see that $\psi$ belongs to $\bar{S}\left(\varphi_{k}, r_{k}\right)$ if and only if

$$
\varphi_{k}(x)-r_{k} \leqq \psi(x) \leqq \varphi_{k}(x)+r_{k}
$$

for all $x$ in $\mathscr{K}$.

Since any two of these spheres intersect, it follows that $\varphi_{k}(x)-r_{k} \leqq$ $\varphi_{l}(x)+r_{l}$ for all $x$ in $\mathscr{H}$, and $1 \leqq k, l \leqq n$. Therefore $\max _{k}\left[\varphi_{k}(x)-r_{k}\right] \leqq$ $\min _{l}\left[\varphi_{l}(x)+r_{l}\right]$ for all $x$ in $\mathscr{K}$. Let $\phi(x)=\max \left[\varphi_{k}(x)-r_{k}\right]$. Clearly $\phi(x)$ is continuous and satisfies (5.1) for every $k$. Hence $\mathscr{C}(\mathscr{C})$ is $\boldsymbol{s}_{0}$ hyperconvex.

In order to characterize the space $\mathscr{H}$ for which $\mathscr{C}(\mathscr{H})$ is $\mathrm{m}$ hyperconvex $\left(\mathrm{n}>\widehat{H}_{0}\right)$, we shall introduce the following property $Q_{\mathfrak{m}}$ :

Definition 1. A Hausdorff space $\mathscr{H}$ is said to satisfy the property $\left(Q_{\mathfrak{m}}\right)$, where $\mathfrak{m}$ is a cardinal $>0$, if for any two classes of open subsets of $\mathscr{L},\left\{\mathscr{Q}_{i}\right\}_{i \in I}$ and $\left\{\mathscr{V}_{j}\right\}_{j \in J}$, such that card $(I)$ and card $(J)<\mathfrak{m}$, $\overline{\mathscr{U}}_{i_{1}} \subset \bigcup_{i \in I} \mathscr{U}_{i}$ and $\overline{\mathscr{V}_{j_{1}}} \subset \bigcup_{j \in J} \mathscr{\mathscr { V }}_{j}$ for any $i_{1} \in I, j_{1} \in J$, and $\left(\bigcup_{i \in I} \mathscr{U}_{i}\right) \cap\left(\bigcup_{j \in J} \mathscr{\mathscr { V }}_{j}\right)=0$, we have $\underset{i \in I}{\mathbb{T}_{i}} \cap \overline{\bigcup_{j \in J} \mathscr{q}_{j}}=0$.

REMARK 1. It is of interest to connect the property $\left(Q_{\mathfrak{n}}\right)$ with some 15 Problem 2 of Nachbin's Paper [14] requires essentially the proof of a converse of this theorem. It seems, therefore, that the theorem was known to Nachbin. 
well known properties. Consider for instance a totally disconnected compact Hausdorff space $\mathscr{C}$. It is well known (Stone's Theorem [16]) that such a space corresponds to a Boolean algebra. Actually it is determined by the Boolean algebra of its subsets which are open and closed. Consider the notion of $m$-completeness ${ }^{16}$ for this Boolean algebra. The m-completeness of this Boolean algebra is equivalent to the fact that $\overline{\bigcup_{i \in I} G_{i}}$ is open for any class $\left\{G_{i}\right\}_{i \in I}$ of open and closed subsets of $\mathscr{H}$ with card $(I)<\mathfrak{m}$. We can state now that the $\mathfrak{m}$-completeness of the Boolean algebra implies the property $\left(Q_{\mathfrak{m}}\right)$ for the corresponding totally disconnected compact Hausdorff space $\mathscr{K}$. In fact, if the classes $\left\{\mathscr{U}_{i}\right\}_{i \in I}$ and $\left\{\mathscr{Y}_{j}\right\}_{j \in I}$ satisfy the requirements of the property $\left(Q_{\mathfrak{m}}\right)$, and $\mathscr{H}$ is totally disconnected, we can replace every set $Z_{i_{1}}$ by an open and closed set $\mathscr{U}_{i_{1}}^{\prime}$ so that $\overline{\mathscr{U}}_{i_{1}} \subset \mathscr{U}_{i_{1}}^{\prime} \subset \bigcup_{i \in I} \mathscr{U}_{i}$. We find similarly an open and closed set $\mathscr{V}_{j_{1}}^{\prime}$ with $\overline{\mathscr{V}}_{j_{1}} \subset \mathscr{V}_{j_{1}}^{\prime} \subset \bigcup_{j \in J} \mathscr{q}_{j}$. Clearly $\bigcup_{i \in I} \mathscr{U}_{i}^{\prime}=\bigcup_{i \in I} \mathscr{U}_{i}$ and $\bigcup_{j \in J} \mathscr{V}_{j}^{\prime}=\bigcup_{j \in J} \mathscr{V}_{j}$. Furthermore $\bigcup_{i \in I} \mathscr{Q}_{i}^{\prime} \cap \bigcup_{j \in J} \mathscr{V}_{j}^{\prime}=0$. But by our hypothesis $\bar{U}_{i} \mathscr{U}_{i}^{\prime}$ is open and closed. Hence we have $\bar{U}_{i} \mathscr{U}_{i}^{\prime} \cap \bar{U}_{j} \mathscr{V}_{j}^{\prime \prime}=0$.

REMARK 2. If a compact Hausdorff space $\mathscr{H}$ has the property $\left(Q_{\mathfrak{m}}\right)$ for $m$ greater than the cardinal of a basis of open sets in $\mathscr{H}$, then $\mathscr{H}$ is extremally disconnected. In fact, if $G$ is any open set in $\mathscr{H}$, consider $G_{1}=\mathscr{H}-\bar{G}$. In a basis of open sets in $\mathscr{H}$ of cardinal $<\mathfrak{m}$, consider all those sets $\mathscr{U}$ such that $\overline{\mathscr{U}} \subset G$, and all those sets $\mathscr{Y}$ such that $\overline{\mathscr{V}} \subset G_{1}$. Clearly card $(\{\mathscr{U}\})$ and card $(\{\mathscr{Y}\})$ are less than $m$. Also the classes $\{\mathscr{U}\}$ and $\{\mathscr{Y}\}$ satisfy all the requirements of the property $\left(Q_{\mathfrak{m}}\right)$ since $\cup \mathscr{\ell}=G$ and $\cup \mathscr{Y}=G_{1}$. Therefore by property $\left(Q_{\mathfrak{m}}\right), \bar{U} /=\bar{G}$ is disjoint from $\overline{U \mathscr{Y}}=\overline{G_{1}}$. It follows that $\overline{G_{1}} \subset \mathscr{C}-\bar{G}=G_{1}$. Hence $G_{1}=\overline{G_{1}}$, and so $\bar{G}=\mathscr{H}-G_{1}$ is open and closed.

We shall need later the following general lemma about compact Hausdorff spaces.

Lemma 1. Let $\varphi$ and $\psi$ be two real finite valued functions defined on a compact Hausdorff space $\mathscr{H}$. In order that there exist a continuous function $f$ defined on $\mathscr{H}$ such that $\varphi(x) \leqq f(x) \leqq \psi(x)$ for every $x$ in $\mathscr{H}$, it is necessary and sufficient that

$$
\limsup _{x^{\prime} \rightarrow x} \varphi\left(x^{\prime}\right) \leqq \liminf _{x^{\prime} \rightarrow x} \phi\left(x^{\prime}\right) .
$$

16 We call here a Boolean algebra m-complete, if for any class of its elements with cardinal less than $\mathfrak{m}$ there exists a least upper bound. This is not the usual notion of $\mathfrak{m}$ completeness, where all subsets of cardinal $\leqq n t$ are accepted. See G. Birkhoff [3]. 
( $\limsup _{x^{\prime} \rightarrow x} \varphi\left(x^{\prime}\right)$ can be defined, by cosidering the class $\mathfrak{U}_{x}$ of all neighborhoods of $x$, as $\inf _{\mathcal{U} \in \mathfrak{H}_{x}} \sup _{x^{\prime} \in \mathcal{V}} \varphi\left(x^{\prime}\right)$. Similarly, $\liminf _{x^{\prime} \rightarrow x} \psi\left(x^{\prime}\right)=\sup _{\mathcal{U} \in \mathfrak{H}_{x}} \inf _{x^{\prime} \in \mathcal{V}} \psi\left(x^{\prime}\right)$.)

REMARK 3. The essential part of this lemma is well known in the case of metric spaces (See F. Hausdorff [7]), and was proved recently in the case of normal spaces (which include compact Hausdorff spaces) by M. Katětov [10], and separately by H. Tong [17]. This essential part is the following theorem: if $\bar{\varphi}$ is upper semicontinuous, $\bar{\psi}$ is lower semicontinuous, and $\bar{\varphi} \leqq \bar{\psi}$, then there exists a continuous function $f$ with $\bar{\varphi} \leqq f \leqq \bar{\psi}$. Our lemma results immediately from this, since (5.2) is clearly necessary for the existence of a continuous $f$ with $\varphi \leqq f \leqq \psi$. On the other hand, denote $\limsup _{x^{\prime} \rightarrow x} \varphi\left(x^{\prime}\right)$ by $\bar{\varphi}(x)$, and $\liminf _{x^{\prime} \rightarrow x} \psi\left(x^{\prime}\right)$ by $\bar{\psi}(x)$. It follows that $\bar{\varphi}$ is upper semicontinuous, and $\bar{\psi}$ is lower semicontinuous. Hence the above theorem gives the sufficiency.

THEOREM 2. In order that the space $\mathscr{C}(\mathscr{Y})$ for compact Hausdorff space $\mathscr{Y}$ be $\mathrm{nt}$-hyperconvex, it is necessary and sufficient that the space $\mathscr{L}$ satisfy the property $\left(Q_{\mathfrak{m}}\right)$.

Proof. For $m \leqq \aleph_{0}$ there is nothing to prove, since Theorem 1 shows that $\mathscr{C}(\mathscr{K})$ is always m-hyperconvex, and it is obvious that the property $\left(Q_{\mathfrak{m}}\right)$ is satisfied by every Hausdorff space.

We shall therefore consider only $m>\kappa_{0}$.

Necessity. Suppose that the property $\left(Q_{\mathfrak{m}}\right)$ is not satisfied. Then there exist two classes $\left\{\mathscr{C}_{i}\right\}_{i \in I}$ and $\left\{\mathscr{V}_{j}\right\}_{j \in J}$, satisfying the requirements of the property $\left(Q_{m}\right)$, such that there exists a point $p$ in $\widetilde{U}_{i} \mathscr{\mathscr { U }}_{i} \cap \widetilde{U}_{j} \mathscr{\mathscr { V }}_{j}$. Put $\mathscr{U}=\bigcup_{i} \mathscr{U}_{i}, \mathscr{V}=\bigcup_{j} \mathscr{V}_{j}$, and $F=\mathscr{H}-(\mathscr{U} \cup \mathscr{V})$. For every $i \in I$ and $j \in J$ we construct a continuous function $\varphi_{i j}(x)$ satisfying the properties:

$$
\begin{aligned}
\varphi_{i j}(x)=0, & x \in \overline{\mathbb{Z}_{i}} ; \\
0 \leqq \varphi_{i j}(x) \leqq \frac{1}{2}, & x \in \mathscr{Q} ; \\
\varphi_{i j}(x)=\frac{1}{2}, & x \in F ; \\
\frac{1}{2} \leqq \varphi_{i j}(x) \leqq 1, & x \in \mathscr{V} ;
\end{aligned}
$$




$$
\varphi_{i j}(x)=1, \quad x \in \overline{\mathscr{V}_{j}} .
$$

Consider now the class of spheres in $\mathscr{C}(\mathscr{C}): \bar{S}\left(\varphi_{i j}, \frac{1}{4}\right), i \in I$ and $j \in J$. Clearly card $(I \times J)<\mathfrak{m}$. These spheres satisfy the requirements of $\mathrm{m}$-hyperconvexity, because

$$
\begin{aligned}
\left|\varphi_{i j}(x)-\varphi_{i_{1} j_{1}}(x)\right| & =0 \quad \text { for } x \in F, \\
& \leqq \frac{1}{2} \quad \text { for } x \in \mathscr{C}, \\
& \leqq \frac{1}{2} \quad \text { for } x \in \mathscr{V},
\end{aligned}
$$

and hence $\left\|\varphi_{i j}-\varphi_{i_{1} j_{1}}\right\| \leqq \frac{1}{2}=\frac{1}{4}+\frac{1}{4}$. By $m$-hyperconvexity, there should exist a continuous function $\varphi$ belonging to all the spheres $\bar{S}\left(\varphi_{i j}, \frac{1}{4}\right)$. This means that $\left|\varphi(x)-\varphi_{i j}(x)\right| \leqq \frac{1}{4}$ for all $(i, j) \in I \times J$ and $x \in \mathscr{H}$. Therefore for $x \in \mathscr{Z}_{i},|\varphi(x)| \leqq \frac{1}{4}$, and for $x \in \mathscr{Y}_{3},|\varphi(x)-1| \leqq \frac{1}{4}$. This being true for all $i$ and $j,|\varphi(x)| \leqq \frac{1}{4}$ for $x \in \mathscr{\ell}$, and $|\varphi(x)-1| \leqq \frac{1}{4}$ for $x$ in $\mathscr{V}$.

Since $\varphi$ is continuous, the former inequality still holds for $x$ in $\overline{\mathscr{U}}$, and the latter for $x$ in $\overline{\mathscr{V}}$. That means the two inequalities hold for the same point $p$, which clearly is impossible. Hence $\mathscr{C}$, for an $m$-hyperconvex space $\mathscr{C}(\mathscr{H})$, satisfies the property $\left(Q_{\mathfrak{m}}\right)$.

Sufficiency. We consider a class of spheres $\bar{S}\left(\chi_{i}, r_{i}\right), \chi_{i} \in \mathscr{C}(\mathscr{H})$, $i \in I$, card $(I)<\mathfrak{m}$, such that

$$
\left\|\chi_{i}-\chi_{j}\right\|=\sup \left[\left|\chi_{i}(x)-\chi_{j}(x)\right|, x \in \mathscr{K}\right] \leqq r_{i}+r_{j}
$$

for any $i, j \in I$. We want to prove that there exists a function $\chi$ in $\mathscr{C}(\mathscr{C})$ such that $\left\|\chi-\chi_{i}\right\| \leqq r_{i}$ for $i \in I$, or equivalently $\chi_{i}(x)-r_{i} \leqq \chi(x) \leqq$ $\chi_{i}(x)+r_{i}$ for all $x \in \mathscr{K}$. From (5.3) we have $\chi_{i}(x)-r_{i} \leqq \chi_{j}(x)+r_{j}$ for all $i, j \in I$, and $x \in \mathscr{K}$. It follows that the functions

$$
\varphi(x)=\sup _{i}\left[\chi_{i}(x)-r_{i}\right] \text { and } \quad \psi(x)=\inf _{j}\left[\chi_{j}(x)+r_{j}\right]
$$

satisfy

$$
\varphi(x) \leqq \psi(x)
$$


Clearly both $\varphi(x)$ and $\psi(x)$ are finite-valued. Define $\bar{\varphi}(x)=\limsup _{x^{\prime} \rightarrow x} \varphi\left(x^{\prime}\right)$, and $\bar{\psi}(x)=\liminf _{x^{\prime} \rightarrow x} \psi\left(x^{\prime}\right)$. It is easy to check that $\bar{\varphi}(x) \leqq \bar{\psi}(x)$ for all $x$ in $\mathscr{H}$ if and only if for any real numbers $\alpha, \beta$, such that $\alpha<\beta$,

$$
\underset{x}{\mathrm{E}}[\varphi(\bar{x})>\beta] \cap \underset{x}{\mathrm{E}}[\bar{\psi}(x)<\alpha]=0 \text {. }
$$

Furthermore,

$$
\underset{x}{\mathrm{E}}[\bar{\varphi}(x)>\beta]=\bigcup_{m=1}^{\infty} \overline{\mathrm{E}} \overline{\left[\varphi\left(x^{\prime}\right)>\beta+\frac{1}{m}\right]},
$$

and

$$
\underset{x}{\mathrm{E}}[\bar{\psi}(x)<\alpha]=\bigcup_{n=1}^{\infty} \overline{\mathrm{E}} \overline{[}\left[\psi\left(x^{\prime}\right)<\alpha-\frac{1}{n}\right]
$$

On the other hand, we have

$$
\underset{x}{\mathrm{E}}\left[\varphi\left(x^{\prime}\right)>\beta+\frac{1}{m}\right]=\bigcup_{i \in I} \underset{x}{\mathrm{E}}\left[\chi_{i}\left(x^{\prime}\right)-r_{i}>\beta+\frac{1}{m}\right]=\bigcup_{i \in I} \mathscr{U}_{i, m},
$$

and

$$
\underset{x}{\mathrm{E}}\left[\psi(x)<\alpha-\frac{1}{n}\right]=\bigcup_{i \in I} \underset{x}{\mathrm{E}}\left[\chi_{j}(x)+r_{j}<\alpha-\frac{1}{n}\right]=\bigcup_{i \in I} \mathscr{Y}_{j, n} .
$$

Since $\chi_{i} \pm r_{i}$ are continuous functions for $i \in I$, the sets $\mathscr{U}_{i, m}$ and $\mathscr{V}_{j, n}$ are open, and so are $\bigcup_{j \in I} \mathscr{C}_{i, m}$ and $\bigcup_{j \in I} \mathscr{V}_{j, n}$. Moreover, card $(I \times N)<\mathfrak{m}$, where $N=\{1,2, \cdots\}$. It is also clear that

$$
\mathbb{Z}_{i_{1}, m_{1}} \subset \underset{x}{\mathrm{E}}\left[\chi_{i_{1}}(x)-r_{i_{1}} \geq \beta+\frac{1}{m_{1}}\right] \subset \mathscr{U}_{i_{1}, m_{1}+1} \subset \bigcup_{i, m} \mathscr{U}_{i, m},
$$

and similarly, $\overline{\mathscr{V}}_{j_{1}, n_{1}} \subset \mathscr{y}_{j_{1}, n_{1}+1} \subset \bigcup_{j, n} \mathscr{y}_{j, n}$. We claim that $\mathscr{U}_{i, m} \cap \mathscr{y}_{j, n}=0$ for every $i, j \in I$ and $m, n \in N$. Suppose this is not true. Then let $x$ be a point in $\ell_{i, m} \cap \%_{j, n}$ for some $i, j, m$ and $n$. It follows that

$$
\chi_{j}\left(x^{\prime}\right)+r_{j}<\alpha-\frac{1}{n}<\alpha<\beta<\beta+\frac{1}{m}<\chi_{i}(x)-r_{i} .
$$

But this contradicts (5.3). Therefore we have $\bigcup_{i, m} \mathscr{C}_{i, m} \cap \bigcup_{j, n} \mathscr{Y}_{j, n}=0$. Hence the two classes $\left\{\mathscr{C}_{i, m}\right\}_{(i, m) \in I \times N}$ and $\left\{\mathscr{y}_{j, n}\right\}_{(j, n) \in I \times N}$ satisfy the requirements of property $\left(Q_{\mathfrak{m}}\right)$, and so we obtain $\bigcup_{i, m} \mathscr{C}_{i, m} \cap \bigcup_{j, n} \psi_{j, n}=0$. Since

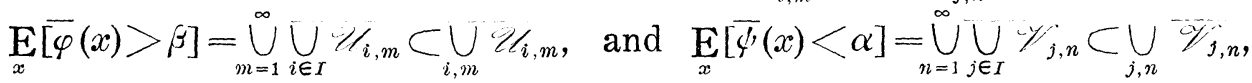
then (5.5) is true, and hence $\bar{\varphi}(x) \leqq \bar{\psi}(x)$ for every $x \in \mathscr{K}$. By Lemma 
1 , there exists a continuous function $\chi$ such that $\bar{\varphi} \leqq \chi \leqq \bar{\psi}$, which implies $\chi_{i}(x)-r_{i} \leqq \chi(x) \leqq \chi_{i}(x)+r_{i}$ for all $x$ in $\mathscr{H}$. Hence the theorem is proved.

REMARK 4. In Remarks 1 and 2 we consider in particular totally disconnected compact Hausdorff spaces $\mathscr{H}$, which therefore correspond to Boolean algebras. In connection with Theorem 2 it is of interest to find the property of the Boolean algebra equivalent to the property $\left(Q_{\mathfrak{m}}\right)$ of the corresponding Hausdorff space $\mathscr{H}$. As we have already noticed in the previous Remarks, we can limit the classes $\left\{\mathbb{Z}_{i}\right\}_{i \in I}$ and $\left\{\mathscr{V}_{j}\right\}_{j \in J}$ in property $\left(Q_{\mathfrak{m}}\right)$ to those composed of only open and closed sets. The thesis of property $\left(Q_{\mathfrak{m}}\right)$ means then that there exists an open and closed subset $G$ of $\mathscr{K}$ such that every $\mathscr{C}_{i} \subset G$ and every $\mathscr{V}_{j} \subset$ $\mathscr{H}-G$. That allows immediate translation into a property $\left(Q^{\prime} \mathfrak{m}\right)$ of the corresponding Boolean algebra $\mathfrak{A}$.

Property $\left(Q^{\prime} \mathfrak{n t}\right)$. For any two subsets $\mathfrak{X}^{\prime}$ and $\mathfrak{U}^{\prime \prime}$ of $\mathfrak{A}$ satisfying

1) $\operatorname{card}\left(\mathfrak{U}^{\prime}\right)<\mathfrak{m}$, card $\left(\mathfrak{Y}^{\prime \prime}\right)<\mathfrak{m}$ and

2) if $a^{\prime} \in \mathfrak{Y ^ { \prime }}$ and $a^{\prime \prime} \in \mathfrak{U}^{\prime \prime}$ then $a^{\prime} \subset a^{\prime \prime}$,

there exists an element $b \in \mathfrak{A}$ such that $a^{\prime} \subset b \subset a^{\prime \prime}$ for all $a^{\prime} \in \mathfrak{X}^{\prime}, a^{\prime \prime} \in \mathfrak{U}^{\prime \prime} .^{17}$

Here elements of $\mathfrak{H}^{\prime}$ correspond to $冖_{i}$, elements of $\mathfrak{X}^{\prime \prime}$ correspond to $\mathscr{K}-\mathscr{Y}_{j}$, and $b$ corresponds to $G$.

6. Examples and problems. By using the theorems of $\S 5$, we are able to construct examples of Banach spaces which are m-hyperconvex, $\mathfrak{n} \geqq \hat{s}_{0}$, but not $\mathfrak{m}^{\prime}$-hyperconvex for $\mathfrak{m}^{\prime}>\mathfrak{m}$. The simplest example of this kind will be the following.

Consider in an abstract set $X$ with card $(X)=\mathfrak{m} \geqq \psi_{0}$, the Boolean algebra $\mathfrak{A}$ of all subsets $A$ of $X$ such that either card $(A)<\mathfrak{m}$ or card $(X-A)<\mathfrak{m}$. This Boolean algebra is $\mathfrak{m}$-complete, but it is not $\mathfrak{m} \mathfrak{t}^{\prime}$-complete for any $\mathfrak{m}^{\prime}>\mathfrak{m}$. By Remark 1 of $\S 5$, the compact Hausdorff space $\mathscr{C}$ corresponding to this Boolean algebra satisfies the property $\left(Q_{\mathfrak{m}}\right)$, and therefore $\mathscr{C}(\mathscr{C})$ is $m$-hyperconvex. On the other hand, $\mathscr{C}$ does not satisfy the property $\left(Q_{\mathfrak{m}^{\prime}}\right)$ for any $\mathfrak{m}^{\prime}>\mathfrak{m}$. To see this we go back to our Boolean algebra $\mathfrak{A}$, and check on the property $\left(Q^{\prime} \mathfrak{n}^{\prime}\right)$ given in Remark 4 of $\S 5$, which is equivalent to property $\left(Q_{\mathfrak{m}^{\prime}}\right)$ for $\mathscr{H}$. In order to see that the property $\left(Q^{\prime} \mathfrak{m}^{\prime}\right)$ is not satisfied in $\mathfrak{A}$, we choose any two disjoint subsets $B$ and $C$ of $X$ such that card $(B)=$ card $(C)=\mathfrak{m}$. We consider the two classes $\mathfrak{H}^{\prime}$ and $\mathfrak{U}^{\prime \prime}$ defined as follows:

$$
\mathfrak{U}^{\prime}=\underset{A^{\prime}}{\mathrm{E}}\left[A^{\prime}=(b), b \in B\right] ; \quad \mathfrak{U}^{\prime \prime}=\underset{A^{\prime \prime}}{\mathrm{E}}\left[A^{\prime \prime}=X-(c), c \in C\right] .
$$

17 This kind of property for general lattices or partially ordered sets has already been encountered in other considerations. See F. Riesz [15], and G. Birkhoff [4]. 
Clearly card $\left(\mathfrak{U}^{\prime}\right)=$ card $\left(\mathfrak{U}^{\prime \prime}\right)=\mathfrak{m}<\mathfrak{m}^{\prime}$. Moreover, $(b) \subset X-(c)$ for every $(b) \in \mathfrak{U}^{\prime}$, and $X-(c) \in \mathfrak{U}^{\prime \prime}$. However, there is no $D \in \mathfrak{A}$ with $(b) \subset$ $D \subset X-(c)$ for all $b \in B, c \in C$. In fact, we would have then $B \subset D$ and $C \subset X-D$, hence card $(D)=\mathfrak{m}$, card $(X-D)=\mathfrak{m}$ contrary to the definition of algebra $\mathfrak{A}$.

For $m<\widehat{s}_{0}$, it is easy to find a 3 -hyperconvex space, which is not 4-hyperconvex. Take, for instance, the Euclidean plane with the natural metric. The following problem is as yet unsolved.

Problem 1. Construct m-hyperconvex spaces (in particular Banach spaces), which are not $(\mathfrak{m}+1)$-hyperconvex for $4 \leqq m<\kappa_{0}$.

In connection with the property $\left(Q^{\prime} \mathfrak{m}\right)$ of the Boolean algebra, which is equivalent to the property $\left(Q_{\mathfrak{m}}\right)$ for the corresponding compact Hausdorff space, we proved, in Remark 1 of $\S 5$, what essentially amounts to the fact that the property $\left(Q^{\prime} \mathrm{m}\right)$ is implied by the m-completeness of the Boolean algebra. The following problem arises.

Problem 2. Are there Boolean algebras which satisfy the property $\left(Q_{\mathfrak{m}}^{\prime}\right)$ for some $\mathrm{m}>\boldsymbol{\aleph}_{0}$, and which are not $\mathrm{m}$-complete?

We do not know of any such example.

Till now we were able to construct compact Hausdorff spaces with property $\left(Q_{\mathfrak{m}}\right)$ only among those which correspond to Boolean algebras, that is, those which are totally disconnected. Therefore there arises another problem.

Problem 3. Is a compact Hausdorff space, satisfying the property $\left(Q_{\mathfrak{m}}\right)$ for $\mathfrak{m}>\widehat{\aleph}_{0}$, necessarily totally disconnected?

It is easy to prove that this is so if the space $\mathscr{C}$ satisfies the first axiom of countability.

L. Nachbin states the following problem:

Is every th-hyperconvex Banach space whose unit sphere has an extreme point necessarily isomorphic to a space $\mathscr{C}(\mathscr{C})$, with a compact Hausdorff $\mathscr{H}$ ?

If the answer to this problem were affirmative, then Theorem 2 of $\S 5$ would imply the following statement: in order that a Banach space with an extreme point in its unit sphere be $\mathrm{m}$-hyperconvex with $\mathrm{m} \geqq \mathrm{s}_{0}$, it is necessary and sufficient that it be isomorphic to the space $\mathscr{C}(\mathscr{C})$ for a compact Hausdorff space $\mathscr{C}$ satisfying property $\left(Q_{\mathfrak{m}}\right)$.

Theorem $1^{\prime}$ of $\S 3$ states that $m$-hyperconvexity for $m>\aleph_{0}$ implies completeness. One may ask if the restriction " $m>w_{0}$ " is necessary. A counter-example for $\mathfrak{m}=\boldsymbol{t}_{0}$ is provided by the linear normed space of all bounded sequences, $\xi=\left\{\xi_{n}\right\}$, such that only a finite number of $\xi_{n}$ are different from zero, with the usual norm, $\|\xi\|=\sup _{n}\left|\xi_{n}\right|$. This space 
is obviously not complete, and it is easy to check that it is s-hyperconvex ${ }^{18}$.

Theorem 4 of $\S 3$ leads to another question: is the almost $(m+1)$ hyperconvexity in its hypothesis necessary or could it be replaced by almost $m$-hyperconvexity. We could obviously replace it for $m \geqq \kappa_{0}$, but for $\mathfrak{m}<\boldsymbol{\aleph}_{0}$ it cannot be done in general. A relevant counter-example for $\mathfrak{m}=3$ is the following:

Consider $^{19}$ in the plane the points $p=(0,0), q=(2,0)$, and $s_{n}=\left(1, \frac{1}{n}\right)$, $n=1,2, \cdots$. The space $\mathscr{E}$ is then $\bigcup_{n=1}^{\infty}\left(\left[p ; s_{n}\right] \cup\left[s_{n} ; q\right]\right)$. On this space, which is not closed in the Euclidean plane, we consider first the Euclidean metric, and then the resulting geodesic metric $\rho^{* 0}$. It is easy to check that in our present space $\mathscr{E}$ the geodesic metric $\rho$ exists, and is complete. Therefore the space $\mathscr{E}$ with the metric $\rho$ is complete and almost 3 -hyperconvex ${ }^{20}$. That it is not 3 -hyperconvex results from the consideration of the two points $p$ and $q$, for which there exists no point $x$ in $\mathcal{E}, x \neq p, x \neq q$, such that $\rho(p, x)+\rho(x, q)=\rho(p, q)$. The following problem however, remains unsolved.

Problem 4. Is there an almost $n$-hyperconvex metric space $\mathscr{E}$, for $3<\mathfrak{m}<\boldsymbol{N}_{0}$, which is complete and not $\mathfrak{n}$-hyperconvex?

Following Theorem $4 \S 3$, an example for this problem with $4<$ $m<\kappa_{0}$ would at the same time give an example for problem 1 and cardinal $\mathrm{n}-1$.

At the end of $\S 3$ we saw that every hyperconvex space is a generalized absolute retract, and if the space is compact, then it is an absolute retract (Corollaries 4 and 5). Here an interesting metrization problem arises.

Problem 5. If a metrizable space $\mathscr{E}$ is a generalized absolute retract, is it possible to define a metric in $\mathscr{S}$, which induces the given topology on $\mathcal{C}$, and makes it into a hyperconvex space?

This problem is of interest even when we restrict ourselves to compact spaces, that is, to absolute retracts.

18 This example was mentioned by L. Nachbin [14].

19 This example was given by N. Aronszajn [1].

20 In a metric space $\varepsilon$ where every two points are connected by an arc of finite length, the geodesic distance between two points $x, y$ is defined to be the infimum of the lengths of all arcs joining $x$ and $y$. Whenever the geodesic metric exists in a space $\mathcal{E}$, the space with this metric is almost 3-hyperconvex. 


\section{REFERENCES}

1. N. Aronszajn, On metric and metrization, Thesis, University of Warsaw, 1930 (unpublished).

2. - Neuer Beweis der Streckenverbundenheit vollständiger konvexer Räume, Ergebnisse eines mathematischen Kolloquiums, (Wien), Heft 6 (1935), 45-46.

3. G. Birkhoff, Lattice theory, Amer. Math. Soc. Colloquium Publications, 25 (1948).

4. — Lattice ordered groups, Ann. of Math. 43 (1942), 298-331.

5. K. Borsuk, Sur les rétractes, Fund. Math. 17 (1931), 152-170.

6. D. B. Goodner, Projections in normed linear spaces, Trans. Amer. Math. Soc. 69 (1950), 89-108.

7. F. Hausdorff, Mengenlehre, 2d. ed., Berlin and Leipzig, 1927.

8. ____._. Erweiterung einer Homöomorphie, Fund. Math. 16 (1930), 353-360.

9. E. Hille, Functional analysis and semi-groups, Amer. Math. Soc. Colloquium Publications, 31 (1948).

10. M. Katětov, On real-valued functions in topological spaces, Fund. Math. 38 (1951), 85-91.

11. J. L. Kelley, Banach spaces with the cxtension property, Trans. Amer. Math. Soc. 72 (1952), 323-326.

12. K. Menger, Untersuchungen über allegemeine Metrik, I, II, III, Math. Ann. 100 (1928), 75-163.

13. Metrische Untersuchungen, Ergebnisse eines mathematischen Kolloquiums, (Wien), Heft 1 (1931), 20-27.

14. L. Nachbin, A Theorem of the Hahn-Banach type, Trans. Amer. Math. Soc. 68 (1950), 28-46.

15. F. Riesz, Sur la théorie générale des opérations linéaires, Ann. of Math. 41 (1940), 174-206.

16. M. H. Stone, Applications of the theory of Boolean rings to general topology, Trans. Amer. Math. Soc. 41 (1937), 375-481.

17. H. Tong, Some characterization of normal and perfectly normal spaces, Duke Math. J. 19 (1952), 289-292.

UNIVERSITY OF KANSAS 



\section{PACIFIC JOURNAL OF MATHEMATICS}

\section{EDITORS}

\author{
H. L. Royden \\ Stanford University \\ Stanford, California \\ E. Hewitt \\ University of Washington \\ Seattle 5 , Washington
}

R. P. Dilworth

California Institute of Technology

Pasadena 4, California

\author{
A. HorN* \\ University of California \\ Los Angeles 24, California
}

\section{ASSOCIATE EDITORS}

\author{
E. F. BECKENBACH \\ C. E. BURGESS \\ H. BUSEMANN \\ H. FEDERER
}

\author{
M. HALL \\ P. R. HALMOS \\ V. GANAPATHY IYER \\ R. D. JAMES
}

\author{
M. S. KNEBELMAN \\ I. NIVEN \\ T. G. OSTROM \\ M. M. SCHIFFER
}

J. J. STOKER

G. SZEKERES

F. WOLF

K. YOSIDA

\section{SPONSORS}

\author{
UNIVERSITY OF BRITISH COLUMBIA \\ CALIFORNIA INSTITUTE OF TECHNOLOGY \\ UNIVERSITY OF CALIFORNIA, BERKELEY \\ UNIVERSITY OF CALIFORNIA, DAVIS \\ UNIVERSITY OF CALIFORNIA, LOS ANGELES \\ UNIVERSITY OF CALIFORNIA, SANTA BARBARA \\ MONTANA STATE UNIVERSITY \\ UNIVERSITY OF NEVADA \\ OREGON STATE COLLEGE \\ UNIVERSITY OF OREGON \\ UNIVERSITY OF SOUTHERN CALIFORNIA
}

STANFORD RESEARCH INSTITUTE

STANFORD UNIVERSITY

UNIVERSITY OF UTAH

WASHINGTON STATE COLLEGE

UNIVERSITY OF WASHINGTON

AMERICAN MATHEMATICAL SOCIETY HUGHES AIRCRAFT COMPANY SHELL DEVELOPMENT COMPANY

Mathematical papers intended for publication in the Pacific Journal of Mathematics should be typewritten (double spaced), and the author should keep a complete copy. Manuscripts may be sent to any of the editors. Manuscripts intended for the outgoing editors should be sent to their successors. All other communications to the editors should be addressed to the managing editor, Alfred Horn at the University of California, Los Angeles 24, California.

50 reprints of each article are furnished free of charge; additional copies may be obtained at cost in multiples of 50 .

The Pacific Journal of Mathematics is published quarterly, in March, June, September, and December. The price per volume (4 numbers) is $\$ 12.00$; single issues, $\$ 3.50$. Back numbers are available. Special price to individual faculty members of supporting institutions and to individual members of the American Mathematical Society: $\$ 4.00$ per volume; single issues, $\$ 1.25$.

Subscriptions, orders for back numbers, and changes of address should be sent to Pacific Journal of Mathematics, c/o University of California Press, Berkeley 4, California.

Printed at Kokusai Bunken Insatsusha (International Academic Printing Co., Ltd.), No. 10, 1-chome, Fujimi-cho, Chiyoda-ku, Tokyo, Japan.

* During the absence of E. G. Straus.

PUBLISHED BY PACIFIC JOURNAL OF MATHEMATICS, A NON-PROFIT CORPORATION COPYRIGHT 1956 BY PACIFIC JOURNAL OF MATHEMATICS 


\section{Pacific Journal of Mathematics}

\section{Vol. 6, No. $3 \quad$ BadMonth, 1956}

Richard Arens and James Eells, Jr., On embedding uniform and topological spaces ............................................ 397

N. Aronszajn and Prom Panitchpakdi, Extension of uniformly continuous transformations and hyperconvex metric spaces .............. 405

Kai Lai Chung and Cyrus Derman, Non-recurrent random walks ........ 441

Harry Herbert Corson, III, On some special systems of equations . . . . . . . . . 449

Charles W. Curtis, On Lie algebras of algebraic linear transformations . . . 453

Isidore Heller, Neighbor relations on the convex of cyclic permutations . . . . 467

Solomon Leader, Convergence topologies for measures and the existence of transition probabilities..................................... 479

D. H. Lehmer, On certain character matrices ...................... 491

Michael Bahir Maschler, Minimal domains and their Bergman kernel function ........................................... 501

Wm. M. Myers, Functionals associated with a continuous transformation ................................... 517

Irving Reiner and Jonathan Dean Swift, Congruence subgroups of matrix groups ....................................... 529

Andrew Sobczyk, Simple families of lines ................... 541

Charles Standish, A class of measure preserving transformations ........ 553 Jeremiah Milton Stark, On distortion in pseudo-conformal mapping ..... 565 